\title{
The Automorphism Group of the Irrational Rotation C*-Algebra
}

\author{
George A. Elliott ${ }^{1}$ and Mikael Rørdam ${ }^{2}$ \\ ${ }^{1}$ Mathematics Institute, University of Copenhagen, DK-2100 Copenhagen $\varnothing$, Denmark, and \\ Department of Mathematics, University of Toronto, Toronto, Canada M5S 1A1 \\ ${ }^{2}$ Department of Mathematics and Computer Science, Odense University, DK-5230 Odense M, \\ Denmark
}

Received March 23, 1992

Dedicated to Huzihiro Araki

\begin{abstract}
The structure of the automorphism group of a simple $\mathrm{C}^{*}$-algebra of real rank zero which is an inductive limit of circle algebras is described. In particular, it is proved that the automorphism group of the irrational rotation $\mathrm{C}^{*}$-algebra, $A_{\theta}$, for any irrational number $\theta$, is an extension of a topologically simple group by $\mathrm{GL}_{2}(\mathbb{Z})$.
\end{abstract}

\section{Introduction}

Let $A$ be a unital $C^{*}$-algebra. The automorphism group $\operatorname{Aut}(A)$ of $A$ decomposes into a series

$$
\overline{\operatorname{Inn}}_{0}(A) \triangleleft \overline{\operatorname{Inn}}(A) \triangleleft \operatorname{Aut}(A)
$$

where $\overline{\operatorname{Inn}}(A)$ is the group of approximately inner automorphisms, and $\overline{\operatorname{Inn}}_{0}(A)$ is the closure of the group of inner automorphisms determined by unitaries connected to 1 .

We shall prove, using an argument closely following a paper by de la Harpe and Skandalis, [HS], that if $A$ is a simple $\mathrm{C}^{*}$-algebra of real rank zero satisfying some extra conditions, then the group $\overline{\operatorname{Inn}}_{0}(A)$ is topologically simple (Corollary 2.4). In particular, $\overline{\operatorname{Inn}}_{0}(A)$ is topologically simple for all simple inductive limits of circle algebras which have real rank zero. It seems likely that $\overline{\operatorname{Inn}}_{0}(A)$ is topologically simple for all simple $\mathrm{C}^{*}$-algebras.

By the classification theorem for inductive limits of circle algebras of real rank zero, [E2], it follows that the quotient group $\operatorname{Aut}(A) / \overline{\operatorname{Inn}}(A)$ is isomorphic to the group of automorphisms of the K-theory of $A$ (Theorem 2.1).

Sections 3 and 4 are concerned with computing the quotient group $\overline{\operatorname{Inn}}(A) / \overline{\operatorname{Inn}}_{0}(A)$ for a $C^{*}$-algebra $A$ which is a simple inductive limit of circle 
algebras of real rank zero. It is proved that $\overline{\operatorname{Inn}}(A) / \overline{\operatorname{Inn}}_{0}(A)$ is isomorphic (as a topological group) to the inverse limit of the discrete groups $\mathrm{K}_{1}(A) / n \mathrm{~K}_{1}(A)$, where $n$ belongs to the directed set of positive integers that divide [1] in $\mathrm{K}_{0}(A)$ (Theorem 4.5). It follows that $\overline{\operatorname{Inn}}(A) / \overline{\operatorname{Inn}_{0}}(A)$ is totally disconnected (Proposition 4.9). The group need not be discrete and is not even locally compact in general (Example 4.12).

These results are illustrated with the following two examples. The irrational rotation algebras $A_{\theta}$, where $\theta \in \mathbb{R} \backslash \mathbb{Q}$, are proved in [EE] to be inductive limits of circle algebras. We have that $\overline{\operatorname{Inn}}\left(A_{\theta}\right)=\overline{\operatorname{Inn}}\left(A_{\theta}\right)$ (see Example 4.7), and the group of automorphisms of the $\mathrm{K}$-theory of $A_{\theta}$ is $\mathrm{GL}_{2}(\mathbb{Z})$ (Example 2.2). Hence the automorphism group of $A_{\theta}$ is an extension

$$
\{1\} \rightarrow \overline{\operatorname{Inn}}\left(A_{\theta}\right) \rightarrow \operatorname{Aut}\left(A_{\theta}\right) \rightarrow \mathrm{GL}_{2}(\mathbb{Z}) \rightarrow\{1\},
$$

and the group of approximately inner automorphisms $\overline{\operatorname{Inn}}\left(A_{\theta}\right)$ is topologically simple. We do not know if this extension splits.

Let $B$ be the Bunce-Deddens algebra. The group of automorphisms of the $\mathrm{K}$-theory of $B$ is $\mathrm{GL}_{1}(\mathbb{Z})=\mathbb{Z} / 2 \mathbb{Z}$ (Example 2.2). The quotient of approximately inner automorphisms $\overline{\operatorname{Inn}}(B) / \overline{\operatorname{Inn}_{0}}(B)$ is isomorphic to the additive group of 2-adic integers $\mathbb{Z}_{2}$ (Example 4.12). Hence $\operatorname{Aut}(B)$ is described by the two extensions,

$$
\begin{gathered}
\{1\} \rightarrow \overline{\operatorname{Inn}}(B) \rightarrow \operatorname{Aut}(B) \rightarrow \mathbb{Z} / 2 \mathbb{Z} \rightarrow\{1\}, \\
\{1\} \rightarrow \overline{\operatorname{Inn}}_{0}(B) \rightarrow \overline{\operatorname{Inn}}(B) \rightarrow \mathbb{Z}_{2} \rightarrow\{1\},
\end{gathered}
$$

and $\overline{\operatorname{Inn}}_{0}(A)$ is topologically simple. The first of these extensions splits.

It is decided exactly when a simple inductive limit of circle algebras $A$ of real rank zero is asymptotically abelian, i.e. possesses a sequence $\left(\alpha_{n}\right)_{n=1}^{\infty}$ of automorphisms such that $\alpha_{n}(x) y-y \alpha_{n}(x) \rightarrow 0$ for all $x, y \in A$ (Corollary 3.13 and Proposition 3.14). The methods behind the proof of this result are similar to those proving the results about the quotient of approximately inner automorphisms. The second named author thanks Nigel Higson for sharing ideas leading to these computations, and for hospitality and support during a visit to Pennsylvania State University in the fall of 1990.

\section{The Automorphism Group}

A circle algebra will here mean a $\mathrm{C}^{*}$-algebra of the form

$$
\bigoplus_{j=1}^{r} \mathrm{M}_{n_{j}}(\mathrm{C}(\mathbb{T})) \simeq\left(\bigoplus_{j=1}^{r} \mathrm{M}_{n_{j}}(\mathbb{C})\right) \otimes \mathrm{C}(\mathbb{T}) .
$$

The spectrum of such an algebra is the disjoint union of $r$ copies of the circle $\mathbb{T}$. Let $A$ be a unital inductive limit of circle algebras, and suppose also that $A$ has real rank zero. Then $A$ belongs to the class of algebras which in [E2] is classified by a K-theory invariant. This invariant is the triple

$$
\left(\mathrm{K}_{0}(A) \oplus \mathrm{K}_{1}(A),\left(\mathrm{K}_{0}(A) \oplus \mathrm{K}_{1}(A)\right)^{+},\left[1_{A}\right]\right),
$$

where $\left(\mathrm{K}_{0}(A) \oplus \mathrm{K}_{1}(A)\right)^{+}$consists of zero and all elements of the form $([p],[u])$, where $p \in A \otimes K$ is a projection and $u \in p(A \otimes K) p$ is unitary. If $A$ is simple, then for each projection $p \in A \otimes K$, every element of $K_{1}(A)$ is of the form $[u]$ for some 
unitary $u \in p(A \otimes K) p$. Hence, for simple $A, \mathrm{~K}_{0}(A) \oplus \mathrm{K}_{1}(A)$ has the strict ordering from $\mathrm{K}_{0}(A)$, and so the $K$-theory invariant is a quadruple,

$$
\left(\mathrm{K}_{0}(A), \mathrm{K}_{0}(A)^{+},\left[1_{A}\right], \mathrm{K}_{1}(A)\right) \text {, }
$$

where $\left(\mathrm{K}_{0}(A), \mathrm{K}_{0}(A)^{+},\left[1_{A}\right]\right)$ is the (usual) dimension group for $A$.

By an automorphism of the $K$-theory of $A$, we shall mean an automorphism of $\mathrm{K}_{0}(A) \oplus \mathrm{K}_{1}(A)$ which preserves the order structure $\left(\mathrm{K}_{0}(A) \oplus \mathrm{K}_{1}(A)\right)^{+}$and the order unit $\left[1_{A}\right]$. Let $\operatorname{Aut}(\mathrm{K}(A))$ denote the group of all such automorphisms.

As usual, $\operatorname{Aut}(A)$ denotes the automorphism group of $A$. Every automorphism $\alpha \in \operatorname{Aut}(A)$ induces an automorphism of K-theory, $\alpha_{*} \in \operatorname{Aut}(\mathrm{K}(A))$. By the classification theorem ([E2], Theorem 7.3 and the remarks preceding it), every automorphism of K-theory lifts to an automorphism of the algebra; that is, the map $\alpha \mapsto \alpha_{*} \in \operatorname{Aut}(\mathrm{K}(A))$ is surjective. The uniqueness part of the classification theorem implies that every automorphism that acts trivially on $\mathrm{K}$-theory is approximately inner ([E2], Theorem 7.4). Let $\operatorname{Inn}(A)$ denote the subgroup of inner automorphisms of $A$, and $\operatorname{Inn}(A)$ its closure (in the topology of pointwise convergence), so that $\overline{\operatorname{Inn}}(A)$ is the group of approximately inner automorphisms. The classification results recalled above can be expressed as follows:

Theorem 2.1 ([E2]). Let $A$ be a unital inductive limit of circle algebras, and suppose that $A$ has real rank zero. Then we have a short exact sequence

$$
\{1\} \rightarrow \overline{\operatorname{Inn}}(A) \rightarrow \operatorname{Aut}(A) \rightarrow \operatorname{Aut}(K(A)) \rightarrow\{1\} .
$$

2.2. Examples. (i) Let $\theta \in \mathbb{R} \backslash \mathbb{Q}$ and denote by $A_{\theta}$ the corresponding irrational rotation algebra. The $\mathrm{K}$-theory of $A_{\theta}$ is

$$
\mathrm{K}_{0}\left(A_{\theta}\right)=\mathbb{Z}+\theta \mathbb{Z} \subseteq \mathbb{R}, \quad \mathrm{K}_{1}\left(A_{\theta}\right)=\mathbb{Z}^{2},
$$

with order unit $\left[1_{A}\right]=1 \in \mathbb{Z}+\theta \mathbb{Z}=K_{0}\left(A_{\theta}\right)$. It is easily seen that all (order and order unit preserving) automorphisms of the K-theory of $A_{\theta}$ must fix each element of $\mathrm{K}_{0}\left(A_{\theta}\right)$. Hence,

$$
\operatorname{Aut}\left(\mathrm{K}\left(A_{\theta}\right)\right)=\operatorname{Aut}\left(\mathrm{K}_{1}\left(A_{\theta}\right)\right)=\mathrm{GL}_{2}(\mathbb{Z}) .
$$

B. Brenken and Y. Watatani ( $[\mathrm{Bk}]$ and $[\mathrm{W}]$ ) found an $\mathrm{SL}_{2}(\mathbb{Z})$ action on $A_{\theta}$ which is a partial lifting of the map

$$
\operatorname{Aut}\left(A_{\theta}\right) \rightarrow \mathrm{GL}_{2}(\mathbb{Z})=\operatorname{Aut}\left(\mathrm{K}\left(A_{\theta}\right)\right) .
$$

It seems less clear (but not unlikely) that all of $\mathrm{GL}_{2}(\mathbb{Z})$ should lift. If that were true, then $\operatorname{Aut}\left(A_{\theta}\right)$ would be the semidirect product of $\overline{\operatorname{Inn}}\left(A_{\theta}\right)$ with $\mathrm{GL}_{2}(\mathbb{Z})$.

(ii) Let $B$ denote the Bunce-Deddens algebra of type $2^{\infty}$. The $K$-theory of $B$ is

$$
\mathrm{K}_{0}(B)=\mathbb{Z}\left[\frac{1}{2}\right] \subseteq \mathbb{R}, \quad \mathrm{K}_{1}(B)=\mathbb{Z},
$$

with order unit $\left[1_{B}\right]=1 \in \mathbb{Z}\left[\frac{1}{2}\right]=K_{0}(B)$. Again, all automorphisms of the $K$ theory of $B$ fix $\mathrm{K}_{0}(B)$ elementwise, and so

$$
\operatorname{Aut}(\mathrm{K}(B))=\operatorname{Aut}\left(\mathrm{K}_{1}(B)\right)=\mathbb{Z} / 2 \mathbb{Z} \text {. }
$$

This action does lift to $\operatorname{Aut}(B)$; that is, there is an automorphism of period two which induces the $\mathbb{Z} / 2 \mathbb{Z}$-action on $K(B)$. (The fixed point algebra of one such automorphism was shown to be an AF-algebra by A. Kumjian in [K].)

(iii) The tensor product $B \otimes B$, where $B$ is as in (ii), is proved in [EG] to be an 
inductive limit of circle algebras. It has $K$-theory

$$
\mathrm{K}_{0}(B \otimes B)=\mathbb{Z}\left[\frac{1}{2}\right] \oplus \mathbb{Z}, \quad \mathrm{K}_{1}(B \otimes B)=\mathbb{Z}\left[\frac{1}{2}\right] \oplus \mathbb{Z}\left[\frac{1}{2}\right],
$$

where $\mathrm{K}_{0}(B \otimes B)$ has the strict ordering from $\mathbb{Z}\left[\frac{1}{2}\right] \subseteq \mathbb{R}$ and has order unit $(1,0) \in \mathbb{Z}\left[\frac{1}{2}\right] \oplus \mathbb{Z}$. The automorphism group of the $\mathrm{K}$-theory of $B \otimes B$ is

$$
\operatorname{Aut}(\mathrm{K}(B \otimes B))=\mathbb{Z} / 2 \mathbb{Z} \oplus \mathrm{GL}_{2}\left(\mathbb{Z}\left[\frac{1}{2}\right]\right) \text {. }
$$

The subgroup $\mathrm{GL}_{2}(\mathbb{Z})$ of this group lifts to $\operatorname{Aut}(B \otimes B)$ by [EG].

We now turn to the structure of the group of approximately inner automorphisms $\overline{\operatorname{Inn}}(A)$. Let $\operatorname{Inn}_{0}(A)$ denote the subgroup of all inner automorphisms determined by unitaries in $A$ which are in $\mathrm{U}_{0}(A)$, the connected component of $\mathrm{U}(A)$ containing 1. Let $\overline{\operatorname{Inn}}_{0}(A)$ denote the closure of $\operatorname{Inn}_{0}(A)$ (in the topology of pointwise convergence). Each of the four groups $\operatorname{Inn}_{0}(A), \overline{\operatorname{Inn}}_{0}(A), \operatorname{Inn}(A)$ and $\overline{\operatorname{Inn}}(A)$ is a normal subgroup of $\operatorname{Aut}(A)$.

It is proved below that $\overline{\operatorname{Inn}}_{0}(A)$ is topologically simple when $A$ is simple. The proof of this involves a variation of a theorem of de la Harpe and Skandalis, [HS], which holds for a broader class of algebras but with a more restricted conclusion. The proof presented below closely follows [HS], but some shortcuts are possible since we aim for a lesser result. First, a lemma.

Lemma 2.3. Let $A$ be an infinite dimensional simple unital $\mathrm{C}^{*}$-algebra of real rank zero which is either purely infinite, or is finite and has the cancellation property for projections and has $\mathrm{K}_{0}(A)$ weakly unperforated. Then

(i) for every non-zero projection $p \in A$ and for every $n \in \mathbb{N}$ there is a unital subalgebra of $p A p$ isomorphic to $\mathrm{M}_{n} \oplus \mathrm{M}_{n+1}$, and

(ii) for every pair of non-zero projections $p, q \in A, q$ is a finite sum of projections in $A$ each unitarily equivalent to a subprojection of $p$.

(The two last assumptions on $A$ referred to in the lemma are the following: For any pair of non-zero projections $e, f \in \mathrm{M}_{n}(A)$, if $[e]=[f]$ in $\mathrm{K}_{0}(A)$, then $e$ and $f$ are unitarily equivalent in $\mathrm{M}_{n}(A)$. If $n g>0$ in $K_{0}(A)$ for some positive integer $n$ and some $g \in \mathrm{K}_{0}(A)$, then $g>0$.)

Proof. Both (i) and (ii) are easily proved for purely infinite simple $\mathrm{C}^{*}$-algebras using results from [C]. Assume that $A$ is a finite simple unital $\mathrm{C}^{*}$-algebra of real rank zero, which has cancellation of projections and has $\mathrm{K}_{0}(A)$ weakly unperforated. To prove (i), upon using the cancellation property, it suffices to show that for every $g \in \mathrm{K}_{0}(A)^{+}$and every $n \in \mathbb{N}$ there are $h_{1}, h_{2} \in \mathrm{K}_{0}(A)^{+}$such that $g=n h_{1}$ $+(n+1) h_{2}$. Since $A$ has real rank zero, it follows from Theorem 3.2 of [E2] (see also $[\mathrm{Z}]$ ) that $\mathrm{K}_{0}(A)$ has the Riesz decomposition property. Hence, by Theorem 3.2 of [E1], there is a sequence $G_{1} \rightarrow G_{2} \rightarrow \cdots$ of ordered groups, each a finite direct sum of groups $\mathbb{Z}$ or $\mathbb{Z} \oplus \mathbb{Z} / k \mathbb{Z}$, the latter ordered by $(m, n)>0$ if $m>0$, such that

$$
\lim _{\rightarrow} G_{j} \cong G
$$

Note that if $g \in \mathbb{Z}$, then $g=n h_{1}+(n+1) h_{2}$ for some strictly positive integers $h_{1}$ and $h_{2}$ if $g \geqq n^{2}+n+1$. 
The assumptions on $A$ imply that $A$ has no minimal projections. Therefore, $g \in \mathrm{K}_{0}(A)^{+}$is a sum of $n^{2}+n+1$ non-zero elements in $\mathrm{K}_{0}(A)^{+}$. Choose $j$ large enough that each of these $n^{2}+n+1$ elements lies in $G_{j}$. Then $g$ has coordinate $\geqq n^{2}+n+1$ in each of the summands of $G_{j}$. Hence $g=n h_{1}+(n+1) h_{2}$ for some $h_{1}, h_{2} \in G^{+}$.

Let $p, q \in A$ be non-zero projections. Because $A$ is simple, $n[p]>[q]$ for some $n \in \mathbb{N}$ (cf. [Bw]). Use (i) to find [q] $=n h_{1}+(n+1) h_{2}$ for some $h_{1}, h_{2} \in \mathrm{K}_{0}(A)^{+}$. It follows that $n[p]>n\left(h_{1}+h_{2}\right)$, and so, because $\mathrm{K}_{0}(A)$ is weakly unperforated, $[p]>h_{1}+h_{2}$ and $[p]>h_{2}$. Thus $[q]$ is a sum of $n+1$ elements of $\mathrm{K}_{0}(A)^{+}$each dominated by $[p]$. Use the cancellation property to complete the proof.

Theorem 2.4. Let $A$ be a simple unital $\mathrm{C}^{*}$-algebra of real rank zero which is either purely infinite, or is finite, has the cancellation property for projections and has $\mathrm{K}_{0}(A)$ weakly unperforated. It follows that the group

$$
U_{0}(A) / \mathbb{T} 1
$$

is topologically simple (i.e. has no non-trivial closed normal subgroups).

Proof. Let $V$ be a closed normal subgroup of $\mathrm{U}_{0}(A)$ not contained in $\mathbb{T} 1$. Let us prove that $V=\mathrm{U}_{0}(A)$. It suffices to show that $V$ contains all exponentials $\exp (i h)$ with $h=h^{*} \in A$. Because $A$ has real rank zero and $V$ is closed it suffices to show that all unitaries with finite spectrum are in $V$, and hence we need only prove that $\lambda p+(1-p) \in V$, where $p \in A$ is a projection and $\lambda \in \mathbb{T}$.

Let $p_{0} \in A$ be a fixed non-zero projection. From Lemma 2.3 every projection in $A$ is a finite sum of projections in $A$ each of which is unitarily equivalent to a subprojection of $p_{0}$. Therefore, to prove that $V=\mathrm{U}_{0}(A)$, it will suffice to show that $\lambda p+(1-p)$ belongs to $V$ for every projection $p \leqq p_{0}$ in $A$, and every $\lambda \in \mathbb{T}$, where $p_{0}$ is some fixed non-zero projection in $A$ (to be specified later).

By assumption, $V$ contains a non-central unitary $u$. Arguing as in Lemma 9.3 of [HS], we may also assume that $\|u-1\|<1 / 2$. It follows that the spectrum of $u$ contains two distinct elements $\lambda_{1}$ and $\lambda_{2}$ with $\left|\lambda_{1}-\lambda_{2}\right| \leqq 2\|u-1\|<1$. Use that each hereditary subalgebra of $A$ contains a non-zero projection ([BP]) to find non-zero orthogonal projections $r_{1}$ and $r_{2}$ in $A$ such that

$$
\left\|u r_{j}-\lambda_{j} r_{j}\right\|<\varepsilon \quad(j=1,2),
$$

where $\varepsilon>0$ will be specified later. When also using simplicity of $A$, one finds non-zero equivalent projections $q_{1}$ and $q_{2}$ in $A$ with $q_{j} \leqq r_{j}$ (use for example [R, Lemma 3.4] and [BP]). Let $p_{0}$ in the paragraph above be $q_{1}$. Let $p_{1} \leqq p_{0}=q_{1}$ be given and find $p_{2} \leqq q_{2}$ equivalent to $p_{1}$. (Recall that we must prove that $\lambda p_{1}+\left(1-p_{1}\right) \in V$ for $\lambda \in \mathbb{T}$.) Notice that

$$
\left\|u p_{j}-\lambda_{j} p_{j}\right\|<\varepsilon \quad(j=1,2) .
$$

Let $s \in A$ be a partial isometry with $s^{*} s=p_{1}$ and $s s^{*}=p_{2}$. Put $z=s+s^{*}$ $+\left(1-p_{1}-\mathrm{p}_{2}\right)$. Choose $\varepsilon>0$ small enough that

$$
\left\|z u z^{*} u^{*}-\left(\bar{\lambda}_{1} \lambda_{2} p_{1}+\bar{\lambda}_{2} \lambda_{1} p_{2}+\left(1-p_{1}-p_{2}\right)\right)\right\|<\frac{1}{2}\left|\lambda_{1}-\lambda_{2}\right| .
$$

Then the spectrum of $z u z^{*} u^{*}$ is contained in three disjoint balls with centres $\bar{\lambda}_{1} \lambda_{2}, \bar{\lambda}_{2} \lambda_{1}$ and 1 . (To see this note that $\left|\lambda_{1}+\lambda_{2}\right| \geqq 1$.) It follows that $z u z^{*} u^{*}=x_{1}+x_{2}+x_{3}$, where $x_{j}^{*} x_{j}=q_{j}=x_{j} x_{j}^{*}$ for projections $q_{j}$ in $A$ with sum 
1 , and $\left\|p_{j}-q_{j}\right\|<1(j=1,2)$ if $\varepsilon>0$ is small enough. Hence $w g_{j} w^{*}=p_{j}(j=1,2)$ for some unitary $w \in U_{0}(A)$. Put

$$
v=w z u z^{*} u^{*} w^{*}=v_{1}+v_{2}+v_{3} \in V,
$$

where $v_{j}^{*} v_{j}=p_{j}=v_{j} v_{j}^{*}(j=1,2)$ and $v_{3}^{*} v_{3}=1-p_{1}-p_{2}=v_{3} v_{3}^{*}$. Then

$$
v z v^{*} z^{*}=v_{1} s^{*} v_{2}^{*} s+v_{2} s v_{1}^{*} s^{*}+\left(1-p_{1}-p_{2}\right) \in V .
$$

Moreover, the spectra of $v_{1} s^{*} v_{2}^{*} s$ and $v_{2} s v_{1}^{*} s^{*}$ are contained in balls with centres $\bar{\lambda}_{1}^{2} \lambda_{2}^{2}$ and $\bar{\lambda}_{2}^{2} \lambda_{1}^{2}$ and radii which are small if $\varepsilon$ is small. Because $\left|\lambda_{1}-\lambda_{2}\right|<1$, the imaginary part of $\bar{\lambda}_{1}^{2} \lambda_{2}^{2}$ is non-zero. We may therefore assume that $\operatorname{Im}\left(\bar{\lambda}_{1}^{2} \lambda_{2}^{2}\right)>0$, and it follows that if $\varepsilon>0$ is small enough, the spectrum of $v_{1} s^{*} v_{2}^{*} s$ relative to $p_{1} A p_{1}$ is contained in $\{\lambda \in \mathbb{T} \mid \operatorname{Im} \lambda>0\}$.

We can now follow the proofs of Lemmas 9.7 and 9.8 in [HS] to find that $\mu p_{1}+\bar{\mu} p_{2}+\left(1-p_{1}-p_{2}\right) \in V$ for some $\mu \in \mathbb{T}, \mu \neq \bar{\mu}$. By (i), for any $n_{0} \in \mathbb{N}$ there is a finite dimensional unital subalgebra $B$ of $\left(p_{1}+p_{2}\right) A\left(p_{1}+p_{2}\right)$ such that $p_{1}$ and $p_{2}$ are equivalent projections in $B$ and such that each direct summand of $B$ is a full matrix algebra of order $\geqq n_{0}$. Because the only proper normal subgroups of $\operatorname{SU}(n)$ are the subgroups of its centre, it follows that the normal subgroup of $U(B)$ generated by $\mu p_{1}+\bar{\mu} p_{2}$ contains an element within $\left|\exp \left(2 \pi i / n_{0}\right)-1\right|$ of $\lambda p_{1}+p_{2}$. Hence $V$ contains an element within $\left|\exp \left(2 \pi i / n_{0}\right)-1\right|$ of $\lambda p_{1}+\left(1-p_{1}\right)$. Since $n_{0} \in \mathbb{N}$ is arbitrary and $V$ is closed, it follows that $\lambda p_{1}+\left(1-p_{1}\right)$ belongs to $V$.

Corollary 2.5. Under the same assumption on $A$ as in Theorem 2.3 it follows that the group $\overline{\operatorname{Inn}}_{0}(A)$ is topologically simple.

Proof. Let $G$ be a closed normal subgroup of $\overline{\operatorname{Inn}}_{0}(A)$ different from $\{1\}$. Set

$$
V=\left\{u \in U_{0}(A) \mid \operatorname{Ad} u \in G\right\} \text {. }
$$

Then $V$ is closed normal subgroup of $U_{0}(A)$. Choose $\alpha \in G$ different from the identity. Then $v^{*} \alpha(v) \notin \mathbb{C} 1$ for some $v \in U_{0}(A)$. Since

$$
(\operatorname{Ad} v)^{-1} \alpha(\operatorname{Ad} v) \alpha^{-1}=\operatorname{Ad} v^{*} \alpha(v)
$$

it follows that $v^{*} \alpha(v) \in V$. Hence, by Theorem 2.3, $V=\mathrm{U}_{0}(A)$ and $G=\overline{\operatorname{Inn}}_{0}(A)$.

2.6. Remarks. All simple unital inductive limits of circle algebras of real rank zero satisfy the assumptions of Theorem 2.4 and Corollary 2.5. Theorem 2.4 and Corollary 2.5 apply more generally, for example, as noted, to all purely infinite simple $\mathrm{C}^{*}$-algebras.

In Sect. 4 the quotient group $\overline{\operatorname{Inn}}(A) / \overline{\operatorname{Inn}}_{0}(A)$ will be described. That, together with Theorem 2.1, describes the automorphism group $\operatorname{Aut}(A)$ modulo the topologically simple group $\overline{\operatorname{Inn}}_{0}(A)$, and modulo the unsettled questions whether the sequence

$$
\{1\} \rightarrow \overline{\operatorname{Inn}}_{0}(A) \rightarrow \overline{\operatorname{Inn}}(A) \rightarrow \overline{\operatorname{Inn}}(A) / \overline{\operatorname{Inn}}_{0}(A) \rightarrow\{1\}
$$

and the sequence in Theorem 2.1 split.

It is conceivable that all unital simple infinite dimensional $\mathrm{C}^{*}$-algebras of real rank zero satisfy the conditions (i) and (ii) considered in Lemma 2.3. Hence Theorem 2.4 and Corollary 2.5 might be valid in that generality. It seems possible that $\overline{\operatorname{Inn}}_{0}(A)$ may be a topologically simple group for every simple $\mathrm{C}^{*}$-algebra $A$, 
but the authors do not know that. An argument by Thomsen [T] using the determinant of de la Harpe and Skandalis shows that the commutator subgroup $\mathrm{U}_{0}(A)^{\prime}$ is not dense in $\mathrm{U}_{0}(A)$ if $A$ does not have real rank zero. Hence $\mathrm{U}_{0}(A) / \mathrm{T} 1$ is not topologically simple when $A$ does not have real rank zero.

Thomsen [T] has extended the results in [HS] and proved that $\mathrm{U}_{0}(A)^{\prime}=\mathrm{U}(A)^{\prime}$ and that this group modulo its centre is algebraically simple for $A$ belonging to a class of simple inductive limit $\mathrm{C}^{*}$-algebras which includes inductive limits of circle algebras. Real rank zero is not assumed in [T].

\section{Asymptotically Abelian Systems}

This section is concerned with constructing embeddings with prescribed K-theory of one circle algebra into another, such that the relative commutant of the image of the first algebra inside the second is large. One application is Theorem 3.7 which

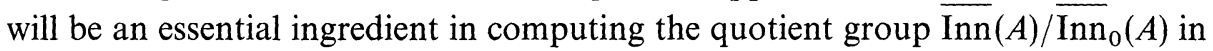
Section 4. As another application it will be decided precisely under what conditions a simple unital inductive limit of circle algebras of real rank zero is asymptotically abelian (Corollary 3.13 and Proposition 3.14).

Let

$$
A_{1}=\bigoplus_{i=1}^{r} \mathrm{M}_{p_{i}}(\mathrm{C}(\mathbb{T})) \quad \text { and } \quad A_{2}=\bigoplus_{j=1}^{s} \mathrm{M}_{q_{j}}(\mathrm{C}(\mathbb{T}))
$$

be two circle algebras, and let $\varphi: A_{1} \rightarrow A_{2}$ be a homomorphism. Then $\varphi$ induces maps

$$
\begin{aligned}
& \mathrm{K}_{0}(\varphi): \mathrm{K}_{0}\left(A_{1}\right) \rightarrow \mathrm{K}_{0}\left(A_{2}\right), \\
& \mathrm{K}_{1}(\varphi): \mathrm{K}_{1}\left(A_{1}\right) \rightarrow \mathrm{K}_{1}\left(A_{2}\right) .
\end{aligned}
$$

Because $\mathrm{K}_{0}\left(A_{1}\right)=\mathbb{Z}^{r}$ and $\mathrm{K}_{0}\left(A_{2}\right)=\mathbb{Z}^{s}$ as ordered abelian groups, with distinguished order units $\left(p_{1}, \ldots, p_{r}\right)$, respectively $\left(q_{1}, \ldots, q_{s}\right)$, the map $\mathrm{K}_{0}(\varphi)$ may be viewed as an $s \times r$ matrix with non-negative integral coefficients which maps $\left(p_{1}, \ldots, p_{r}\right)$ to $\left(q_{1}, \ldots, q_{s}\right)$. We have $\mathrm{K}_{1}\left(A_{1}\right)=\mathbb{Z}^{r}$ and $\mathrm{K}_{1}\left(A_{2}\right)=\mathbb{Z}^{s}$ as abelian groups, and so $\mathrm{K}_{1}(\varphi)$ is an $s \times r$ matrix with (possibly negative) integral coefficients.

The embeddings of the circle algebras will be composed from familiar examples described below.

Amplification. The embedding of amplification by a factor $n \in \mathbb{N}$,

$$
\mu_{n}: A \rightarrow \mathrm{M}_{n} \otimes A,
$$

where $A$ is any $\mathrm{C}^{*}$-algebra, is the map

$$
\mu_{n}(a)=1_{n} \otimes a \quad(a \in A) .
$$

Note that $\mathrm{K}_{0}\left(\mu_{n}\right)=\mathrm{K}_{1}\left(\mu_{n}\right)=n$ (multiplication by $n$ ).

$n$-times around embedding. Let $n \in \mathbb{Z} \backslash\{0\}$ and consider the homomorphism

$$
\lambda_{n}: \mathrm{C}(\mathbb{T}) \rightarrow \mathrm{M}_{|n|}(\mathrm{C}(\mathrm{T})) .
$$


that maps the generator $z$ of $\mathrm{C}(\mathrm{T})$ into

$$
\left(\begin{array}{ccccc}
0 & z & & & \\
& 0 & 1 & & \\
& & \ddots & \ddots & \\
& & & \ddots & 1 \\
1 & & & & 0
\end{array}\right) \text { if } n>0, \quad\left(\begin{array}{ccccc}
0 & \bar{z} & & & \\
& \ddots & 1 & & \\
& & \ddots & \ddots & \\
& & & \ddots & 1 \\
1 & & & & 0
\end{array}\right) \text { if } n<0
$$

We have $\mathrm{K}_{0}\left(\lambda_{n}\right)=|n|$ and $\mathrm{K}_{1}\left(\lambda_{n}\right)=n /|n|$.

Standard homomorphism. A homomorphism

$$
\varphi: \mathrm{M}_{p}(\mathrm{C}(\mathbb{T})) \rightarrow \mathrm{M}_{q}(\mathrm{C}(\mathbb{T}))
$$

is called standard if it is the composition of homomorphisms

$$
\begin{aligned}
\mathrm{M}_{p} \otimes \mathrm{C}(\mathbb{T}) & \rightarrow \bigoplus_{j} \mathrm{M}_{a_{j}} \otimes \mathrm{M}_{p} \otimes \mathrm{M}_{\left|b_{j}\right|} \otimes \mathrm{C}(\mathbb{T}) \\
& \rightarrow \mathrm{M}_{q} \otimes \mathrm{C}(\mathbb{T}),
\end{aligned}
$$

where $a_{j} \in \mathbb{N}, b_{j} \in \mathbb{Z} \backslash\{0\}$, the first homomorphism is

$$
\bigoplus_{j} \mu_{a_{j}} \circ\left(\mathrm{id}_{p} \otimes \lambda_{b_{j}}\right)
$$

and the second homomorphism is $l \otimes \mathrm{id}_{\mathrm{C}(\mathbb{T})}$, where $l$ is a homomorphism of finite dimensional algebras that maps one-dimensional projections into one-dimensional projections. The composed map $\mathrm{M}_{p}(\mathrm{C}(\mathrm{T})) \rightarrow \mathrm{M}_{q}(\mathrm{C}(\mathrm{T}))$ will be written

$$
\varphi=\sum_{j} \mu_{a_{j}} \circ\left(\mathrm{id}_{p} \otimes \lambda_{b_{j}}\right) \text {. }
$$

If all $b_{j} \in \mathbb{Z} \backslash\{-1,0,1\}$, then $\varphi$ is called strictly winding. Note that

$$
\begin{aligned}
& \mathrm{K}_{0}(\varphi)=\sum_{j} a_{j}\left|b_{j}\right|, \\
& \mathrm{K}_{1}(\varphi)=\sum_{j} a_{j} b_{j} /\left|b_{j}\right| .
\end{aligned}
$$

A homomorphism

$$
\varphi: \bigoplus_{i=1}^{r} \mathrm{M}_{p_{i}}(\mathrm{C}(\mathbb{T})) \rightarrow \bigoplus_{j=1}^{s} \mathrm{M}_{q_{j}}(\mathrm{C}(\mathbb{T}))
$$

is called a (strictly winding) standard homomorphism if each of the partial homomorphisms

$$
\mathrm{M}_{p_{i}}(\mathrm{C}(\mathrm{T})) \rightarrow \mathrm{M}_{q_{j}}(\mathrm{C}(\mathrm{T}))
$$

is either a (strictly winding) standard homomorphism or zero.

The following result is easily deduced from [BBEK].

Proposition 3.1. Let

$$
A_{1} \stackrel{\varphi_{1}}{\longrightarrow} A_{2} \stackrel{\varphi_{2}}{\longrightarrow} A_{3} \rightarrow \cdots
$$


be a sequence of circle algebras. If each of the connecting homomorphisms $\varphi_{j}: A_{j} \rightarrow A_{j+1}$ is a strictly winding standard homomorphism, then the inductive limit $\mathrm{C}^{*}$-algebra has real rank zero.

It will be necessary to single out a certain subclass of the standard homomorphisms, the usefulness of which will follow from 3.3 to 3.7 .

3.2. Definition. A standard homomorphism $\varphi: A_{1} \rightarrow A_{2}$ between circle algebras is said to have property (SK) if for each summand $\mathrm{M}_{p}(\mathrm{C}(\mathrm{T}))$ of $A_{1}$ and $\mathrm{M}_{q}(\mathrm{C}(\mathrm{T})$ ) of $A_{2}$, the partial homomorphism of $\mathrm{M}_{p}(\mathrm{C}(\mathbb{T}))$ into $\mathrm{M}_{q}(\mathrm{C}(\mathrm{T}))$ is of the form

$$
\sum_{k=1}^{t} \mathrm{id}_{p} \otimes \lambda_{a_{k}},
$$

where the integers $\left(a_{k}\right)_{k=1}^{t}$ are relatively prime.

Lemma 3.3. The composition of two standard homomorphisms with property (SK) will also have property (SK).

Proof. This follows from the facts that the composition of two $n$-times around embeddings is given as

$$
\lambda_{m \cdot n}=\left(\mathrm{id}_{|n|} \otimes \lambda_{n}\right) \circ \lambda_{m}
$$

and that the family of integers $\left(a_{k} b_{l}\right)$ is relatively prime if the families $\left(a_{k}\right)$ and $\left(b_{l}\right)$ are relatively prime.

Lemma 3.4. Let $\varphi: A_{1} \rightarrow A_{2}$ be a unital standard homomorphism with property (SK) between circle algebras $A_{1}$ and $A_{2}$. Write $A_{1}=\mathrm{M}_{n} \otimes B_{1}$, where

$$
B_{1}=\bigoplus_{i=1}^{r} \mathrm{M}_{p_{i}}(\mathrm{C}(\mathrm{T}))
$$

and the family of integers $\left(p_{i}\right)$ is relatively prime. Then the inclusion $\varphi\left(A_{1}\right)^{\prime} \cap A_{2} \subseteq A_{2}$ induces a map

$$
\mathrm{K}_{1}\left(\varphi\left(A_{1}\right)^{\prime} \cap A_{2}\right) \rightarrow \mathrm{K}_{1}\left(A_{2}\right)
$$

the image of which is $n \mathrm{~K}_{1}\left(A_{2}\right)$.

Proof. It suffices to consider the case that $A_{2}=\mathrm{M}_{q}(\mathrm{C}(\mathrm{T}))$. Then $\varphi$ has the form

$$
\begin{aligned}
A_{1} & =\bigoplus_{i=1}^{r} \mathrm{M}_{n p_{i}} \otimes \mathrm{C}(\mathbb{T}) \\
& \rightarrow \bigoplus_{i=1}^{r} \bigoplus_{j=1}^{t_{i}} \mathrm{M}_{n p_{l}} \otimes \mathrm{M}_{\left|a_{i j}\right|} \otimes \mathrm{C}(\mathbb{T}) \\
& \rightarrow \mathrm{M}_{q} \otimes \mathrm{C}(\mathrm{T})=A_{2},
\end{aligned}
$$

and the commutant of $A_{1}$ relative to the intermediate algebra is

$$
\bigoplus_{i=1}^{r} \bigoplus_{j=1}^{t_{1}} 1_{n p_{i}} \otimes 1_{\left|a_{i j}\right|} \otimes C(\mathbb{T}) \text {. }
$$


For every $k=1, \ldots, r$ and $l=1, \ldots, t_{k}$ let $u_{k l} \in \varphi\left(A_{1}\right)^{\prime} \cap A_{2}$ denote the unitary operator which is the image in $A_{2}$ of

$$
\bigoplus_{i=1}^{r} \bigoplus_{j=1}^{t_{i}} 1_{n p_{i}} \otimes 1_{\left|a_{i j}\right|} \otimes f_{i j}
$$

where $f_{i j}=1$ if $(i, j) \neq(k, l)$, and $f_{k l}(z)=z \quad(z \in \mathbb{T})$. Then $\left[u_{k l}\right]=n p_{k} a_{k l} \in \mathbb{Z}$ $=\mathrm{K}_{1}\left(A_{2}\right)$. By the hypothesis on the integers $p_{k}$ and $a_{k l}$, the integers $n p_{k} a_{k l}$ generate the group $n \mathbb{Z}=n \mathrm{~K}_{1}(A)$.

Lemma 3.5. Let $D_{0}=\left(\alpha_{i j}\right)$ and $D_{1}=\left(\beta_{i j}\right)$ be $r \times s$ matrices with integral coefficients satisfying

$$
\alpha_{i j} \geqq \max \left\{13,3\left|\beta_{i j}\right|\right\}
$$

for all $i, j$. Let $p_{1}, \ldots, p_{\mathbf{r}}$ be positive integers, and set

$$
\left(\begin{array}{c}
q_{1} \\
\vdots \\
q_{s}
\end{array}\right)=D_{0}\left(\begin{array}{c}
p_{1} \\
\vdots \\
p_{r}
\end{array}\right) .
$$

Then there is a strictly winding standard homomorphism with property (SK),

$$
\varphi: \bigoplus_{i=1}^{r} \mathrm{M}_{p_{i}}(\mathrm{C}(\mathrm{T})) \rightarrow \bigoplus_{j=1}^{s} \mathrm{M}_{q_{j}}(\mathrm{C}(\mathrm{T})),
$$

with $\mathrm{K}_{0}(\varphi)=D_{0}$ and $\mathrm{K}_{1}(\varphi)=D_{1}$.

Proof. It suffices to consider the case $r=s=1$. We must then find

$$
\varphi: \mathrm{M}_{p}(\mathrm{C}(\mathrm{T})) \rightarrow \mathrm{M}_{q}(\mathrm{C}(\mathrm{T}))
$$

of the desired type, such that $\mathrm{K}_{0}(\varphi)=\alpha$ and $\mathrm{K}_{1}(\varphi)=\beta$ when $\alpha \geqq \max \{13,3|\beta|\}$. One can find integers $a_{1}, a_{2}, \ldots, a_{t} \in\{2,3\}$ and $\varepsilon_{1}, \varepsilon_{2}, \ldots, \varepsilon_{t} \in\{-1,1\}$ with $a_{1}=2, a_{2}=3$,

$$
\alpha=\sum_{k=1}^{t} a_{k}, \quad \text { and } \quad \beta=\sum_{k=1}^{t} \varepsilon_{k} .
$$

Then

$$
\varphi=\sum_{k=1}^{t} \mathrm{id}_{p} \otimes \lambda_{\varepsilon_{k} a_{k}}
$$

has the desired properties.

Lemma 3.6. Let $A$ be a unital simple $C^{*}$-algebra of real rank zero which is an inductive limit of circle algebras. Let positive integers $v_{0}=v_{0}\left(v_{1}, C\right)$ be given for each non-negative integer $v_{1}$ and each circle algebra $C$. Then $A$ can be written as the inductive limit of a sequence of circle algebras

$$
A_{1} \stackrel{\varphi_{1}}{\longrightarrow} A_{2} \stackrel{\varphi_{2}}{\longrightarrow} A_{3} \stackrel{\varphi_{3}}{\longrightarrow} \cdots
$$

in which the connecting maps $\varphi_{j}: A_{j} \rightarrow A_{j+1}$ are such that if $v_{1}$ is the maximum of the absolute values of the entries in $\mathrm{K}_{1}\left(\varphi_{j}\right)$, then each entry of $\mathrm{K}_{0}\left(\varphi_{j}\right)$ is greater than 
$v_{0}\left(v_{1}, A_{j}\right)$. The connecting maps $\varphi_{j}$ can be chosen to be strictly winding standard homomorphisms with property (SK).

Proof. Let $A$ be written as the inductive limit of a sequence of circle algebras

$$
B_{1} \stackrel{\psi_{1}}{\longrightarrow} B_{2} \stackrel{\psi_{2}}{\longrightarrow} B_{3} \longrightarrow \cdots \text {. }
$$

Replacing the given function $v_{0}=v_{0}\left(v_{1}, C\right)$ by a larger one, we may suppose that

$$
v_{0}\left(v_{1}, C\right) \geqq \max \left\{13,3 v_{1}\right\} \text {. }
$$

Let $r_{j}$ denote the number of summands in $B_{j}$, so that $\mathrm{K}_{0}\left(B_{j}\right)=\mathbb{Z}^{r_{j}}=\mathrm{K}_{1}\left(B_{j}\right)$. Upon passing to a subsequence of $(*)$ we may assume that $r_{1} \leqq r_{2} \leqq r_{3} \leqq \cdots$. The image in $A$ of each minimal projection in $B_{j}$ is full in $A$ because $A$ is simple. By a standard argument there is an $i>j$ such that the image in $B_{i}$ of each minimal projection in $B_{j}$ is full in $B_{i}$. By passing to a subsequence of $(*)$ we may suppose that $i=j+1$. In that case, each entry of $K_{0}\left(\psi_{j}\right)$ is non-zero. Note that the smallest entry in the matrix

$$
\mathrm{K}_{0}\left(\psi_{i} \psi_{i-1} \cdots \psi_{j}\right)=\mathrm{K}_{0}\left(\psi_{i}\right) \mathrm{K}_{0}\left(\psi_{i-1}\right) \cdots \mathrm{K}_{0}\left(\psi_{j}\right)
$$

tends to infinity as for each fixed $j, i>j$ tends to infinity.

Passing to a subsequence of $(*)$, we may suppose that each entry in $\mathrm{K}_{0}\left(\psi_{j}\right)$ is greater than $v_{0}\left(v_{1}, B_{j}\right)$, where $v_{1}=0$ if $j=1$, and $v_{1}$ is the maximum of the absolute values of the entries in $\mathrm{K}_{1}\left(\psi_{j-1}\right)$ if $j \geqq 2$.

By Lemma 3.5 there are strictly winding standard homomorphisms $\varphi_{j}: A_{j} \rightarrow A_{j+1}$ with property (SK) and with

$$
\mathrm{K}_{0}\left(\varphi_{j}\right)=\mathrm{K}_{0}\left(\psi_{j}\right), \quad \mathrm{K}_{1}\left(\varphi_{j}\right)=D_{j},
$$

where $D_{1}=0$, and $D_{j}$ is the $r_{j+1} \times r_{j}$ matrix which has the $r_{j} \times r_{j-1}$ matrix $\mathrm{K}_{1}\left(\varphi_{j-1}\right)$ in its upper left corner and zeros elsewhere when $j \geqq 2$. By Proposition 3.1 the inductive limit

$$
A_{1} \stackrel{\varphi_{1}}{\longrightarrow} A_{2} \stackrel{\varphi_{2}}{\longrightarrow} A_{3} \stackrel{\varphi_{3}}{\longrightarrow} \cdots \longrightarrow \bar{A}
$$

has real rank zero. By construction, $\bar{A}$ has the same $\mathrm{K}$-theory invariant (for simple algebras) as $A$, and so, by the classification theorem ([E2]), $\bar{A}$ is isomorphic to $A$.

Theorem 3.7. Let $A$ be a unital simple $\mathrm{C}^{*}$-algebra of real rank zero which is an inductive limit of circle algebras. Then $A$ is the inductive limit of a sequence of circle algebras

$$
A_{1} \rightarrow A_{2} \rightarrow A_{3} \rightarrow \cdots
$$

such that, if $n_{j}$ denotes the largest integer for which there is a decomposition $A_{j}=\mathrm{M}_{n_{j}} \otimes B_{j}$, then each connecting map $\varphi_{i j}: A_{j} \rightarrow A_{i}(i>j)$ is such that the map

$$
\mathrm{K}_{1}\left(\varphi_{i j}\left(A_{j}\right)^{\prime} \cap A_{i}\right) \rightarrow \mathrm{K}_{1}\left(A_{i}\right)
$$

has image equal to $n_{j} \mathrm{~K}_{1}\left(A_{i}\right)$.

Proof. This is an immediate consequence of Lemmas 3.3, 3.4 and 3.6.

A $C^{*}$-algebra $A$ will be said to be asymptotically abelian, with respect to a sequence $\left(\alpha_{n}\right)$ of automorphisms, if

$$
\alpha_{n}(x) y-y \alpha_{n}(x) \rightarrow 0
$$


for all $x, y \in A$. We shall establish the existence of such sequences of automorphisms, and, moreover, inner automorphisms, for a large class of simple inductive limits of circle algebras of real rank zero. The proof is along the same lines as the proof of Theorem 3.7, but involves a little more arithmetic. The main step is to prove the following proposition (an analogue of Lemmas 3.4 and 3.5).

Proposition 3.8. Let $p_{1}, \ldots, p_{r}$ be positive integers with gcd equal to $n \in \mathbb{N}$. Then for each $v_{1} \in \mathbb{N}$ there is $v_{0} \in \mathbb{N}$ (depending on $v_{1}$ and on $p_{1}, \ldots, p_{r}$ ) such that the following holds: Let $D_{0}$ and $D_{1}$ be $s \times r$ matrices with integral coefficients divisible by $n$, such that each entry of $D_{1}$ has absolute value $\leqq v_{1}$, and each entry of $D_{0}$ is $\geqq v_{0}$. Set

$$
\left(\begin{array}{c}
q_{1} \\
\vdots \\
q_{s}
\end{array}\right)=D_{0}\left(\begin{array}{c}
p_{1} \\
\vdots \\
p_{r}
\end{array}\right) .
$$

Then there is a strictly winding standard homomorphism

$$
\varphi: \bigoplus_{i=1}^{r} \mathrm{M}_{p_{i}}(\mathrm{C}(\mathbb{T}))=A_{1} \rightarrow \bigoplus_{j=1}^{s} \mathrm{M}_{q_{j}}(\mathrm{C}(\mathbb{T}))=A_{2}
$$

with $\mathrm{K}_{0}(\varphi)=D_{0}$ and $\mathrm{K}_{1}(\varphi)=D_{1}$, and there is a symmetry $u \in A_{2}$ such that

$$
u \varphi\left(A_{1}\right) u^{*} \subseteq \varphi\left(A_{1}\right)^{\prime} \cap A_{2} .
$$

The proof of this proposition depends on solving some Diophantine equations, which is done in the following lemmas.

Lemma 3.9. Let $p_{1}, \ldots, p_{r}$ be positive integers with gcd equal to $n \in \mathbb{N}$.

(i) For each $v_{0}^{\prime} \in \mathbb{N}$ there is $v_{0} \in \mathbb{N}$ such that if $\alpha_{1}, \ldots, \alpha_{r}$ are integers divisible by $n$ and greater than $v_{0}$, then there are integers $\gamma_{i j} \geqq v_{0}^{\prime}(i, j=1, \ldots, r)$ with $\gamma_{i j}=\gamma_{j i}$ and

$$
\alpha_{j}=\sum_{i=1}^{r} \gamma_{i j} p_{i} .
$$

(ii) For each $v_{1} \in \mathbb{N}$ there is $v_{1}^{\prime} \in \mathbb{N}$ such that if $\beta$ is an integer divisible by $n$ and $|\beta| \leqq v_{1}$, then there are integers $\delta_{i}(i=1, \ldots, r)$ with $\left|\delta_{i}\right| \leqq v_{1}^{\prime}$ and

$$
\beta=\sum_{i=1}^{r} \delta_{i} p_{i} .
$$

Proof. For both (i) and (ii) it suffices to consider the case that $n=1$.

(i) Let $\left(e_{1}, \ldots, e_{r}\right)$ be the standard basis for the additive group $\mathbb{Z}^{r}$, and set

$$
t_{i j}=p_{i} e_{j}+p_{j} e_{i} \quad \text { if } i \neq j, \quad \text { and } t_{i i}=p_{i} e_{i} .
$$

It suffices to prove that the elements $\left(t_{i j}\right)_{i, j=1}^{r}$ generate $\mathbb{Z}^{r}$, since it follows from this that

$$
\left\{\sum_{1 \leqq i \leqq j \leqq r} \gamma_{i j} t_{i j} \mid \gamma_{i j} \geqq v_{0}^{\prime}\right\}
$$

contains all elements $\left(\alpha_{1}, \ldots, \alpha_{n}\right) \in \mathbb{Z}^{r}$ with $\alpha_{j} \geqq v_{0}$ for some large enough $v_{0}$. 
For every pair $i \neq j$, note that the subgroup spanned by the elements $\left(t_{i i}, t_{j j}, t_{i j}\right)$ contains

$$
\left(p_{i}, p_{j}\right) e_{i} \text { and }\left(p_{i}, p_{j}\right) e_{j},
$$

where $\left(p_{i}, p_{j}\right)$ is the $\mathrm{gcd}$ of $p_{i}$ and $p_{j}$. The hypothesis that $p_{1}, \ldots, p_{r}$ are relatively prime implies that for each $j$, the integers

$$
\left(p_{1}, p_{j}\right),\left(p_{2}, p_{j}\right), \ldots,\left(p_{r}, p_{j}\right)
$$

are relatively prime. It follows that each $e_{j}$ belongs to the group spanned by $\left(t_{i j}\right)_{i, j=1}^{r}$.

(ii) This is just the definition of $p_{1}, \ldots, p_{r}$ being relatively prime (except for the bounds on $\left|\delta_{i}\right|$, which are trivial to obtain).

Lemma 3.10. For each $v_{1}^{\prime} \in \mathbb{N}$ there is $v_{0}^{\prime} \in \mathbb{N}$ such that (i) and (ii) below hold.

(i) For all integers $\gamma, \delta_{1}$ and $\delta_{2}$ satisfying

$$
\left|\delta_{j}\right| \leqq v_{1}^{\prime} \text { and } \gamma \geqq v_{0}^{\prime},
$$

there are integers $t>0, b_{1 k}, b_{2 k} \in\{2,3\}$, and $\varepsilon_{1 k}, \varepsilon_{2 k} \in\{-1,1\}(k=1, \ldots, t)$ such that

$$
\gamma=\sum_{k=1}^{t} b_{1 k} b_{2 k}, \quad \delta_{j}=\sum_{k=1}^{t} \varepsilon_{j k} b_{j k} \quad(j=1,2) .
$$

(ii) For all integers $\gamma$ and $\delta$ satisfying

$$
|\delta| \leqq v_{1}^{\prime} \text { and } \gamma \geqq v_{0}^{\prime},
$$

there are integers $t>0, a_{k} \in\{2,3\}$, and $\varepsilon_{k} \in\{-1,1\}$ such that

(e) if $\gamma \equiv \delta(\bmod 2)$, then

$$
\gamma=\sum_{k=1}^{t} a_{k}^{2}, \quad \delta=\sum_{k=1}^{t} \varepsilon_{k} a_{k},
$$

(o) and if $\gamma \neq \equiv \delta(\bmod 2)$, then

$$
\gamma=\sum_{k=1}^{t} a_{k}^{2}+12, \quad \delta=\sum_{k=1}^{t} \varepsilon_{k} a_{k}+5 .
$$

Proof. (i) Let $S$ denote the subsemigroup of the additive semigroup $\mathbb{N}$ consisting of all elements of the form

$$
\sum_{k=1}^{t} b_{1 k} b_{2 k}
$$

where $b_{1 k}, b_{2 k} \in\{2,3\}$, and where

$$
\sum_{k=1}^{t} \varepsilon_{1 k} b_{1 k}=0=\sum_{k=1}^{t} \varepsilon_{2 k} b_{2 k}
$$

for suitable choices of $\varepsilon_{j k} \in\{-1,1\}$. Check that $S$ contains the numbers $8,12,18$ and 25 (which generate $\mathbb{Z}$ as a group) and hence that $S$ contains all sufficiently large integers. (In fact, $S$ contains all integers $\geqq 40$.) 
For each pair $\left(\delta_{1}, \delta_{2}\right)$ find (as is easily done) integers $t>0, b_{1 k}, b_{2 k} \in\{2,3\}$, and $\varepsilon_{1 k}, \varepsilon_{2 k} \in\{-1,1\}$ such that

$$
\delta_{1}=\sum_{k=1}^{t} \varepsilon_{1 k} b_{1 k} \quad \text { and } \quad \delta_{2}=\sum_{k=1}^{t} \varepsilon_{2 k} b_{2 k} .
$$

Now take the maximum of the numbers

$$
\sum_{k=1}^{t} b_{1 k} b_{2 k}
$$

arising in this way with $\left(\delta_{1}, \delta_{2}\right)$ such that $\left|\delta_{1}\right|,\left|\delta_{2}\right| \leqq v_{1}^{\prime}$. Add this maximum to the number (40) found in the previous paragraph, and require that $v_{0}^{\prime}$ be greater than this sum.

(ii) It suffices to solve for the even case (e) (since the odd case (o) follows from the even case - possibly after increasing $v_{0}^{\prime}$ ). Accordingly, let us assume that $\gamma \equiv \delta$. We shall follow the same strategy as in the proof of (i) and let $T$ denote the subsemigroup of $\mathbb{N}$ consisting of all elements of the form

$$
\sum_{k=1}^{t} a_{k}^{2}
$$

where $a_{k} \in\{2,3\}$, and where

$$
\sum_{k=1}^{t} \varepsilon_{k} a_{k}=0
$$

for suitable choices of $\varepsilon_{k} \in\{-1,1\}$. Check that $T$ contains the numbers 8 and 18 (which generate $2 \mathbb{Z}$ as a group). Hence $T$ contains all sufficiently large even numbers (in fact, $T$ contains all even integers $\geqq 48$ ).

For each integer $\delta$, find integers $a_{k} \in\{2,3\}$ and $\varepsilon_{k} \in\{-1,1\}$ such that

$$
\delta=\sum_{k=1}^{t} \varepsilon_{k} a_{k} .
$$

Take the maximum of the numbers

$$
\sum_{k=1}^{t} a_{k}^{2}
$$

arising this way as $\delta$ takes all values in $\left\{-v_{1}^{\prime}, \ldots, v_{1}^{\prime}\right\}$, and add the number (48) from the last paragraph to this maximum. Then (ii) (e) holds if $v_{0}^{\prime}$ is greater than this sum.

Proof of Proposition 3.8. Let $v_{1} \in \mathbb{N}$ be given, and let $v_{1}^{\prime} \in \mathbb{N}$ be in Lemma 3.9 (iii). Let next $v_{0}^{\prime} \in \mathbb{N}$ be as in Lemma 3.10 (corresponding to the given $v_{1}^{\prime}$ ), and let finally $v_{0} \in \mathbb{N}$ be as in Lemma 3.9 (i).

It suffices to consider the case that

$$
A_{2}=\mathrm{M}_{q}(\mathrm{C}(\mathrm{T})) \text {. }
$$

Then $D_{0}=\left(\alpha_{1}, \ldots, \alpha_{r}\right), D_{1}=\left(\beta_{1}, \ldots, \beta_{r}\right)$, and

$$
\alpha_{j} \geqq v_{0}, \quad\left|\beta_{j}\right| \leqq v_{1} .
$$


Use Lemma 3.9 to find integers $\gamma_{i j}$ and $\delta_{i j}(i, j=1, \ldots, r)$ with

$$
\begin{aligned}
\gamma_{i j} \geqq v_{0}^{\prime}, \quad\left|\delta_{i j}\right| \leqq v_{1}^{\prime}, \quad \gamma_{i j} & =\gamma_{j i}, \\
\alpha_{j} & =\sum_{i=1}^{r} \gamma_{i j} p_{i}, \quad \text { and } \quad \beta_{j}=\sum_{i=1}^{r} \delta_{i j} p_{i} .
\end{aligned}
$$

Let $1 \leqq i<j \leqq r$ be fixed. Use Lemma 3.10 to find integers $t(i, j), a_{i j k}, a_{j i k}, \varepsilon_{i j k}$ and $\varepsilon_{j i k}(k=1, \ldots, t(i, j))$ with

$$
\begin{aligned}
& a_{i j k}, a_{j i k} \in\{2,3\}, \quad \varepsilon_{i j k}, \varepsilon_{j i k} \in\{-1,1\}, \\
& \gamma_{i j}=\sum_{k=1}^{t(i, j)} a_{i j k} a_{j i k}\left(=\gamma_{j i}\right), \\
& \delta_{i j}=\sum_{k=1}^{t(i, j)} \varepsilon_{i j k} a_{i j k}, \quad \delta_{j i}=\sum_{k=1}^{t(i, j)} \varepsilon_{j i k} a_{j i k} .
\end{aligned}
$$

Let $I_{0}$ denote the set of $i \in\{1, \ldots, n\}$ with $\gamma_{i i} \not \equiv \delta_{i i}(\bmod 2)$. From Lemma 3.10 it follows that there are integers $t(i, i), a_{i i k} \in\{2,3\}$ and $\varepsilon_{i i k} \in\{-1,1\}(k=1, \ldots, t(i, i))$ with

$$
\gamma_{i i}=\sum_{k=1}^{t(i, i)} a_{i i k}^{2}, \quad \delta_{i i}=\sum_{k=1}^{t(i, i)} \varepsilon_{i i k} a_{i i k}
$$

if $i \notin I_{0}$, and

$$
\gamma_{i i}=\sum_{k=1}^{t(i, i)} a_{i i k}^{2}+12, \quad \delta_{i i}=\sum_{k=1}^{t(i, i)} \varepsilon_{i i k} a_{i i k}+5
$$

if $i \in I_{0}$.

The partial homomorphism $\varphi_{j}: \mathrm{M}_{p_{j}} \otimes \mathrm{C}(\mathrm{T}) \rightarrow A_{2}$ is given by

$$
\begin{aligned}
\varphi_{j}= & \sum_{i=1}^{r} \sum_{k=1}^{t(i, j)} \mu_{p_{i} \cdot a_{i j k}} \circ\left(\mathrm{id}_{p_{j}} \otimes \lambda_{\varepsilon_{i j k} \cdot a_{j i k}}\right) \\
& \left(+\mu_{2 p_{j}} \circ\left(\mathrm{id}_{p_{j}} \otimes \lambda_{3}\right)+\mu_{3 p_{j}} \circ\left(\mathrm{id}_{p_{j}} \otimes \lambda_{2}\right)\right),
\end{aligned}
$$

where the summands in parenthesis are included if $j \in I_{0}$. We have

$$
\begin{aligned}
& \mathrm{K}_{0}\left(\varphi_{j}\right)=\sum_{i=1}^{r} \sum_{k=1}^{t(i, j)} p_{i} a_{j i k}\left(+12 p_{j}\right)=\sum_{i=1}^{r} \gamma_{i j} p_{i}=\alpha_{j}, \\
& \mathrm{~K}_{1}\left(\varphi_{j}\right)=\sum_{i=1}^{r} \sum_{k=1}^{t(i, j)} p_{i} a_{i j k} \varepsilon_{i j k}\left(+5 p_{j}\right)=\sum_{i=1}^{r} \delta_{i j} p_{i}=\beta_{j} .
\end{aligned}
$$

Let $\varphi: A_{1} \rightarrow A_{2}$ denote the homomorphism of which the partial homomorphisms are $\varphi_{1}, \ldots, \varphi_{r}$. Then $\mathrm{K}_{0}(\varphi)=D_{0}$ and $\mathrm{K}_{1}(\varphi)=D_{1}$. The relative commutant $\varphi\left(A_{1}\right)^{\prime} \cap A_{2}$ is contained in a subalgebra of $A_{2}$ isomorphic to

$$
\begin{gathered}
\bigoplus_{j=1}^{r} \bigoplus_{i=1}^{r} \bigoplus_{k=1}^{t(i, j)} \mathrm{M}_{p_{i}} \otimes \mathrm{M}_{a_{i j k}} \otimes 1_{p_{j}} \otimes 1_{a_{j i k}} \otimes \mathrm{C}(\mathrm{T}) \\
\oplus \bigoplus_{j \in I_{0}}\left[\left(\mathrm{M}_{p_{j}} \otimes \mathrm{M}_{2} \otimes 1_{p_{j}} \otimes 1_{j}\right) \oplus\left(\mathrm{M}_{p_{j}} \otimes \mathrm{M}_{j} \otimes 1_{p_{j}} \otimes 1_{2}\right)\right] \otimes \mathrm{C}(\mathrm{T}) .
\end{gathered}
$$


The existence of a symmetry $u \in A_{2}$ with $u \varphi\left(A_{1}\right) u^{*} \subseteq \varphi\left(A_{1}\right)^{\prime} \cap A_{2}$ follows from the symmetry between the algebra displayed above and the image of $A_{1}$ in $A_{2}$.

Theorem 3.11. Let $A$ be a simple unital $\mathrm{C}^{*}$-algebra of real rank zero, which is an inductive limit of circle algebras. Suppose that if $n$ is a positive integer that divides [1] in $\mathrm{K}_{0}(A)$, then $n$ divides every element of both $\mathrm{K}_{0}(A)$ and $\mathrm{K}_{1}(A)$. Then $A$ is the inductive limit of a sequence of circle algebras

$$
A_{1} \stackrel{\varphi_{1}}{\longrightarrow} A_{2} \stackrel{\varphi_{2}}{\longrightarrow} A_{3} \stackrel{\varphi_{3}}{\longrightarrow} \cdots,
$$

such that the connecting maps $\varphi_{j}: A_{j} \rightarrow A_{j+1}$ are unital homomorphisms, and

$$
u_{j} \varphi_{j}\left(A_{j}\right) u_{j}^{*} \subseteq \varphi_{j}\left(A_{j}\right)^{\prime} \cap A_{j+1}
$$

for some symmetry $u_{j} \in A_{j+1}$.

Proof. To every circle algebra

$$
\mathrm{C}=\bigoplus_{j=1}^{r} \mathrm{M}_{p_{j}}(\mathrm{C}(\mathrm{T}))
$$

and every $v_{1} \in \mathbb{N}$ associate a positive integer $v_{0}$ as follows. With $n$ being the gcd of $p_{1}, \ldots, p_{r}$, let $v_{0}=v_{0}\left(C, v_{1}\right)$ be as in Proposition 3.8 corresponding to the given $p_{1}, \ldots, p_{r}$, but with $n v_{1}$ in place of $v_{1}$.

Next use Lemma 3.6 to write $A$ as the inductive limit of a sequence of circle algebras

$$
A_{1} \stackrel{\psi_{1}}{\longrightarrow} A_{2} \stackrel{\psi_{2}}{\longrightarrow} A_{3} \longrightarrow \cdots
$$

such that each entry of $\mathrm{K}_{0}\left(\psi_{j}\right)$ is greater than $v_{0}\left(v_{1}, A_{j}\right)$, where $v_{1}$ is the maximum of the absolute values of the entries in $\mathrm{K}_{1}\left(\psi_{j}\right)$. Let $n_{j}$ denote the greatest positive integer dividing [1] in $\mathrm{K}_{0}\left(A_{j}\right)$. By the hypothesis on $\mathrm{K}_{0}(A)$, we may assume that the limit $(*)$ in the proof of Lemma 3.6, on which $(\dagger)$ is built, satisfies that if $n$ divides [1] in $\mathrm{K}_{0}\left(B_{j}\right)$, then $n$ divides $\mathrm{K}_{0}\left(\psi_{j}\right)$. By the proof of Lemma 3.6 we may therefore assume that $n_{j}$ divides $\mathrm{K}_{0}\left(\psi_{j}\right)$ in the limit $(\dagger)$ above.

From Proposition 3.8 and the choice of $v_{0}=v_{0}\left(v_{1}, C\right)$, there are strictly winding standard homomorphisms $\varphi_{j}: A_{j} \rightarrow A_{j+1}$ with

$$
\mathrm{K}_{0}\left(\varphi_{j}\right)=\mathrm{K}_{0}\left(\psi_{j}\right), \quad \mathrm{K}_{1}\left(\varphi_{j}\right)=n_{j} \mathrm{~K}_{1}\left(\psi_{j}\right),
$$

such that $u_{j} \varphi_{j}\left(A_{j}\right) u_{j}^{*} \subseteq \varphi_{j}\left(A_{j}\right)^{\prime} \cap A_{j+1}$ for some symmetry $u_{j} \in A_{j+1}$. By the assumption on $\mathrm{K}_{1}(A)$,

$$
\mathrm{K}_{1}(A)=\mathrm{K}_{1}(A) \underset{\mathbb{Z}}{\otimes} \mathbb{Z}\left[1 / n_{j}\right]
$$

and so the inductive limit, $\bar{A}$, of the sequence

$$
A_{1} \stackrel{\varphi_{1}}{\longrightarrow} A_{2} \stackrel{\varphi_{2}}{\longrightarrow} A_{3} \stackrel{\varphi_{3}}{\longrightarrow} \cdots
$$

has the same K-theory invariant as $A$. Since $\bar{A}$ has real rank zero (Proposition 3.1), the classification theorem ([E2]) yields that $\bar{A}$ is isomorphic to $A$. The proof is complete. 
3.12. Remark. The K-theory condition of Theorem 3.11 is equivalent to the following condition on the algebra: If $n$ is a positive integer, and $A$ has a unital subalgebra isomorphic to $\mathrm{M}_{n}$, then $A$ is isomorphic to $A \otimes \mathrm{M}_{n^{\infty}}$, where $\mathrm{M}_{n^{\infty}}$ is the UHF-algebra of type $n^{\infty}$.

Corollary 3.13. Let $A$ be a $\mathrm{C}^{*}$-algebra satisfying the conditions in Theorem 3.11. It follows that there is a sequence of inner automorphisms $\left(\alpha_{k}\right)_{k=1}^{\infty}$ of $A$, determined by symmetries in A, such that

$$
\alpha_{k}(x) y-y \alpha_{k}(x) \rightarrow 0
$$

for all $x, y \in A$.

Proof. Let $v_{k}$ denote the image in $A$ of the symmetry $u_{k} \in A_{k}$ in Theorem 3.11 , and set $\alpha_{k}=\operatorname{Ad} v_{k}$. Then

$$
\alpha_{k}(x) y=y \alpha_{k}(x)
$$

for all $x$ and $y$ in the image in $A$ of $A_{k-1}$.

The K-theory conditions on $A$ to ensure the asymptotic abelianness are also necessary, as shown below. (See also Remark 3.12).

Proposition 3.14. Let $A$ be a unital $\mathrm{C}^{*}$-algebra, and assume that $A$ is asymptotically abelian with respect to a sequence of (not necessarily inner) automorphisms $\left(\alpha_{k}\right)_{k=1}^{\infty}$ of $A$. Then for each $n \geqq 2$, if $\mathrm{M}_{n}$ is a unital subalgebra of $A$, then $A$ is isomorphic to $A \otimes \mathrm{M}_{n^{\infty}}$.

Proof. Assume that $\mathbf{M}_{n}$ is a unital subalgebra of $A$, and let $\left(e_{i j}\right)_{i, j=1}^{n}$ be a system of matrix units for $A$. Then

$$
\left(\alpha_{k}\left(e_{i j}\right)\right)_{i, j=1}^{n}
$$

is a central sequence of systems of $n \times n$ matrix units for $A$. It follows from [BKR, Proposition 2.12] that $A$ is isomorphic to $A \otimes \mathrm{M}_{n^{\infty}}$.

\section{Approximately Inner Automorphisms}

Let $A$ be a simple unital $\mathrm{C}^{*}$-algebra of real rank zero which is an inductive limit of circle algebras. The four groups of automorphisms $\operatorname{Inn}(A), \overline{\operatorname{Inn}}(A), \overline{\operatorname{Inn}}_{0}(A)$ and $\overline{\operatorname{Inn}}_{0}(A)$ are as in Section 2. Recall that $\overline{\operatorname{Inn}}_{0}(A)$ is a normal subgroup of $\overline{\operatorname{Inn}}(A)$, and that $\operatorname{Inn}_{0}(A)$ is topologically simple if $A$ is simple. We shall compute the quotient group $\overline{\operatorname{Inn}}(A) / \overrightarrow{\operatorname{Inn}}_{0}(A)$.

Lemma 4.1. There is a group homomorphism $\mathrm{K}_{1}(A) \rightarrow \overline{\operatorname{Inn}}(A) / \overline{\operatorname{Inn}}_{0}(A)$ which makes the following diagram with exact rows commute:

$$
\begin{aligned}
& \{1\} \rightarrow \mathrm{U}_{0}(A) \rightarrow \mathrm{U}(A) \rightarrow \mathrm{K}_{1}(A) \rightarrow\{0\} \\
& \{1\} \rightarrow \overline{\operatorname{Inn}}_{0}(A) \rightarrow \overline{\text { Ad }} \rightarrow \overline{\operatorname{Ad}} \frac{\downarrow}{\operatorname{Inn}(A)} \rightarrow \overline{\operatorname{Inn}(A) / \operatorname{Inn}_{0}(A) \rightarrow\{0\}}
\end{aligned}
$$

Proof. Because $A$ is an inductive limit of circle algebras the top row is exact. (It suffices to check this for $A=\mathrm{C}(\mathbb{T})$.) The existence of the map $\mathrm{K}_{1}(A) \rightarrow \overline{\operatorname{Inn}}(A) / \overline{\operatorname{Inn}}_{0}(A)$ now follows from a diagram chase. 
It follows from the diagram that $\overline{\operatorname{Inn}}(A) / \overline{\operatorname{Inn}}_{0}(A)$ is abelian (cf. Lemma 4.2). This group and $\mathrm{K}_{1}(A)$ will both be written additively. Let $\hat{g}$ denote the image of $g \in \mathrm{K}_{1}(A)$ in $\overline{\operatorname{Inn}}(A) / \overline{\operatorname{Inn}}_{0}(A)$ under the map given in Lemma 4.1.

Lemma 4.2. The image of $\mathrm{K}_{1}(A)$ in $\overline{\operatorname{Inn}}(A) / \overline{\operatorname{Inn}}_{0}(A)$ is dense. If $\left(g_{k}\right)$ is a sequence in $\mathrm{K}_{1}(A)$, then

$$
\hat{g}_{k} \rightarrow 0
$$

in $\overline{\operatorname{Inn}}(A) / \overline{\operatorname{Inn}}_{0}(A)$ if and only if there is a central sequence $\left(u_{k}\right)$ of unitaries in $A$ with $\left[u_{k}\right]=g_{k}$.

Proof. The first assertion follows from the diagram in Lemma 4.1. By the definition of the quotient topology, $\hat{g}_{k} \rightarrow 0$ if and only if the sequence lifts to a sequence in $\operatorname{Inn}(A)$ that converges to the identity. Let $\alpha_{k}$ be a lifting of $\hat{g}_{k}$ such that $\alpha_{k} \rightarrow 1$. Then $\alpha_{k}=\beta_{k}$ Ad $u_{k}$ with $u_{k}$ a unitary in $A$ with $\left[u_{k}\right]=g_{k}$, and $\beta_{k} \in \operatorname{Inn}_{0}(A)$. Because the topological group $\overline{\operatorname{Inn}}_{0}(A)$ is first countable, there is a sequence $\left(\gamma_{k}\right)$ in $\operatorname{Inn}_{0}(A)$ such that $\beta_{k} \gamma_{k}^{-1} \rightarrow 1$. Hence $\gamma_{k} \operatorname{Ad} u_{k} \rightarrow 1$ and $\gamma_{k} \operatorname{Ad} u_{k}$ is a lifting of $\hat{g}_{k}$. Absorbing $\gamma_{k}$ into Ad $u_{k}$ we have that Ad $u_{k}$ lifts $\hat{g}_{k},\left[u_{k}\right]=g_{k}$, and Ad $u_{k} \rightarrow 1$. Finally, Ad $u_{k} \rightarrow 1$ if and only if the sequence $\left(u_{k}\right)$ is central in $A$ (i.e., $u_{k} x-x u_{k} \rightarrow 0$ for all $x \in A$ ). This completes the proof.

Lemma 4.2 implies that $\hat{g}=0$ in $\overline{\operatorname{Inn}}(A) / \overline{\operatorname{Inn}}_{0}(A)$ if and only if there is a central sequence $\left(u_{k}\right)$ of unitaries in $A$ with $\left[u_{k}\right]=g$ for all $k$.

4.3. The topology on the image of $\mathrm{K}_{1}(A)$ in $\overline{\operatorname{Inn}}(A) / \overline{\operatorname{Inn}}_{0}(A)$ is given by data from the group $\mathrm{K}_{0}(A)$. More specifically, the data needed from $\mathrm{K}_{0}(A)$ is the directed set of positive integers that divide [1] in $\mathrm{K}_{0}(A)$. (This is the same as the set of positive integers $n$ such that $\mathrm{M}_{n}$ is a unital subalgebra of $A$.) It will be convenient to represent this set by (any) sequence $\left(n_{j}\right)$ of positive integers satisfying

(i) $n_{j} \mid[1]$ in $\mathrm{K}_{0}(A)$,

(ii) $n_{j} \mid n_{j+1}$,

(iii) if $n \mid[1]$ in $\mathrm{K}_{0}(A)$, then $n \mid n_{j}$ for some $j$.

An appropriate choice of sequence $\left(n_{j}\right)$ sastisfying these conditions is the sequence of $n_{j}$ from Theorem 3.7. If there is a greatest integer that divides [1] in $\mathrm{K}_{0}(A)$, then we may take the sequence to be constant equal to that integer.

By construction of $\left(n_{j}\right)$ there is a sequence of surjections

$$
\mathrm{K}_{1}(A) / n_{1} \mathrm{~K}_{1}(A) \leftarrow \mathrm{K}_{1}(A) / n_{2} \mathrm{~K}_{1}(A) \leftarrow \mathrm{K}_{1}(A) / n_{3} \mathrm{~K}_{1}(A) \leftarrow \cdots,
$$

which gives an inverse limit group

$$
\lim \mathrm{K}_{1}(A) / n_{j} \mathrm{~K}_{1}(A)
$$

Equip each of the groups $\mathrm{K}_{1}(A) / n_{j} \mathrm{~K}_{1}(A)$ with the discrete topology, and give the inverse limit group the natural topology from the inverse limit. Let

$$
\pi_{i}: \lim \mathrm{K}_{1}(A) / n_{j} \mathrm{~K}_{1}(A) \rightarrow \mathrm{K}_{1}(A) / n_{i} \mathrm{~K}_{1}(A)
$$


denote the canonical projection. Then $x_{k} \rightarrow x$ in the inverse limit if and only if $\pi_{i}\left(x_{k}\right) \rightarrow \pi_{i}(x)$ for all $i \in \mathbb{N}$, which happens if and only if for all $i \in \mathbb{N}$, $\pi_{i}\left(x_{k}\right) \in n_{i} \mathrm{~K}_{1}(A)+\pi_{i}(x)$ eventually.

Since the quotient maps $\mathrm{K}_{1}(A) \rightarrow \mathrm{K}_{1}(A) / n_{j} \mathrm{~K}_{1}(A)$ commute with the surjections in the inverse limit, they lift to a map

$$
\mathrm{K}_{1}(A) \rightarrow \lim \mathrm{K}_{1}(A) / n_{j} \mathrm{~K}_{1}(A)
$$

Denote by $\check{g}$ the image in the inverse limit of $g$ in $\mathrm{K}_{1}(A)$. Part of the discussion above is then summarized in the lemma below.

Lemma 4.4. The image of $\mathrm{K}_{1}(A)$ in $\lim \leftarrow \mathrm{K}_{1}(A) / n_{j} \mathrm{~K}_{1}(A)$ is dense. Let $\left(g_{k}\right)$ be a sequence in $\mathrm{K}_{1}(A)$. Then

$$
\check{g}_{k} \rightarrow 0
$$

in $\lim \leftarrow \mathrm{K}_{1}(A) / n_{j} \mathrm{~K}_{1}(A)$ if and only if for all $i \in \mathbb{N}$ the sequence $\left(g_{k}\right)$ lies in $n_{i} \mathrm{~K}_{1}(A)$ eventually.

The theorem below, which is the main result of this paper, tells us that the topological groups $\overline{\operatorname{Inn}}(A) / \overline{\operatorname{Inn}}_{0}(A)$ and $\lim \leftarrow \mathrm{K}_{1}(A) / n_{j} \mathrm{~K}_{1}(A)$ are isomorphic.

Theorem 4.5. Let $A$ be a unital simple $\mathrm{C}^{*}$-algebra of real rank zero which is an inductive limit of circle algebras. Then there is an isomorphism of topological groups

$$
\begin{gathered}
\overline{\operatorname{Inn}}(A) / \overline{\operatorname{Inn}_{0}}(A) \leftrightarrow \lim _{\nwarrow} \mathrm{K}_{1}(A) / n_{j} \mathrm{~K}_{1}(A) \\
\mathrm{K}_{1}(A)
\end{gathered}
$$

commuting with the two inclusions of $\mathrm{K}_{1}(A)$.

Proof. Notice first that the two topological groups $\overline{\operatorname{Inn}}(A) / \overline{\operatorname{Inn}}_{0}(A)$ and $\lim _{\leftarrow} \mathrm{K}_{1}(A) / n_{j} \mathrm{~K}_{1}(A)$ are complete. Hence, by Lemmas 4.2 and 4.4 , they are the completions of the respective images of $\mathrm{K}_{1}(A)$. It suffices therefore to prove that these images give the same topology on $\mathrm{K}_{1}(A)$. In other words, it must be shown that for every sequence $\left(g_{k}\right)$ in $\mathrm{K}_{1}(A)$,

$$
\begin{gathered}
\hat{g}_{k} \rightarrow 0 \text { in } \overline{\operatorname{Inn}}(A) / \overline{\operatorname{Inn}}_{0}(A) \\
\check{g}_{k} \rightarrow 0 \text { in } \lim \mathrm{K}_{1}(A) / n_{j} \mathrm{~K}_{1}(A) .
\end{gathered}
$$

" $\Downarrow ":$ Assume that $\hat{g}_{k} \rightarrow 0$. Then, by Lemma 4.2, there is a central sequence $\left(u_{k}\right)$ of unitaries in $A$ with $\left[u_{k}\right]=g_{k}$. Let $l \in \mathbb{N}$. By Lemma 4.4 we must show that $\left(g_{k}\right)$ is in $n_{l} \mathrm{~K}_{1}(A)$ eventually. Let

$$
\left(e_{i j}\right)_{i, j=1}^{n_{l}}
$$

be a system of matrix units for a unital subalgebra of $A$ isomorphic to $\mathrm{M}_{n_{l}}$ (see 4.3 for the existence of this). If $k$ is large, then $u_{k}$ approximately commutes with each of the matrix units $e_{i j}$. In that case,

$$
e_{i i} u_{k} e_{i i}+\left(1-e_{i i}\right)
$$


is close to a unitary $v_{i} \in A$. Moreover, $\left[v_{i}\right]=\left[v_{j}\right]$ in $\mathrm{K}_{1}(A)$, and $u_{k}$ is close to $v_{1} v_{2} \cdots v_{n_{l}}$. Hence, for large $k$,

$$
\left[u_{k}\right]=n_{l}\left[v_{1}\right] \in n_{l} \mathrm{~K}_{1}(A) .
$$

" $\Uparrow$ ": Assume that $\check{g}_{k} \rightarrow 0$. Let $A$ be expressed as an inductive limit of a sequence of circle algebras as in Theorem 3.7. By Lemma 4.2, it will suffice to prove that for each $i \in \mathbb{N}$, if $k \in \mathbb{N}$ is large enough, then $g_{k}=\left[u_{k}\right]$ for some unitary $u_{k} \in A$ which commutes with the image of the algebra $A_{i}$ in $A$.

Let $i \in \mathbb{N}$ be given. By Lemma 4.4 the sequence $\left(g_{k}\right)$ is in $n_{i} \mathrm{~K}_{1}(A)$ eventually. Let $g \in n_{i} \mathrm{~K}_{1}(A)$. Then $g=n_{i} h$ for some $h \in \mathrm{K}_{1}(A)$ in the image of $\mathrm{K}_{1}\left(A_{j}\right) \rightarrow \mathrm{K}_{1}(A)$ for some $j>i$. Hence, by Theorem 3.7, $g$ is in the image of the composed map

$$
\mathrm{K}_{1}\left(\varphi_{j i}\left(A_{i}\right)^{\prime} \cap A_{j}\right) \rightarrow \mathrm{K}_{1}\left(A_{j}\right) \rightarrow \mathrm{K}_{1}(A) .
$$

It follows that $g=[u]$ for some unitary $u$ in the image of $\varphi_{j i}\left(A_{i}\right)^{\prime} \cap A_{j}$ in $A$, and so $u$ commutes with the image of $A_{i}$ in $A$.

From here on, $A$ will be assumed to satisfy the conditions of Theorem 4.5.

Corollary 4.6. The group of approximately inner automorphisms $\overline{\operatorname{Inn}}(A)$ coincides with the group $\overline{\operatorname{Inn}}_{0}(A)$ if and only if $n$ divides each element of $\mathrm{K}_{1}(A)$ for every positive integer $n$ that divides $[1]$ in $\mathrm{K}_{0}(A)$.

Proof. The inverse limit $\lim _{\leftarrow} \mathrm{K}_{1}(A) / n_{j} \mathrm{~K}_{1}(A)$ is zero if and only if $\mathrm{K}_{1}(A)=n_{j} \mathrm{~K}_{1}(A)$ for all $j$.

Example 4.7. For the irrational rotation algebra $A_{\theta}$ (see Example 2.2 (i)), 1 is the only positive integer dividing [1] in $\mathrm{K}_{0}\left(A_{\theta}\right)$. It follows from Corollary 4.6 that $\overline{\operatorname{Inn}}\left(A_{\theta}\right)=\overline{\operatorname{Inn}}_{0}\left(A_{\theta}\right)$. In particular, the canonical $\mathbb{T}^{2}$-action on $A_{\theta}$ (that multiplies the two generators by scalars of modulus 1) can be approximated by inner automorphisms determined by unitaries connected to 1 . Combining this with Theorem 2.4 we see that the group of approximately inner automorphisms $\overline{\operatorname{Inn}}\left(A_{\theta}\right)$ is topologically simple.

Remark 4.8. Let $A$ be any $\mathrm{C}^{*}$-algebra that is asymptotically abelian with respect to a sequence $\left(\alpha_{n}\right)$ of inner automorphisms (see 3.13). Then $\overline{\operatorname{Inn}}(A)=\overline{\operatorname{Inn}}_{0}(A)$. Indeed, for every unitary $u$ in $A, v_{n}=u \alpha_{n}\left(u^{*}\right)$ is in $\mathrm{U}_{0}(A)$ and

$$
\operatorname{Ad} v_{n} \rightarrow \operatorname{Ad} u \text {. }
$$

Hence $\operatorname{Inn}(A) \subseteq \overline{\operatorname{Inn}}_{0}(A)$.

It may happen, even for inductive limits of circle algebras with real rank zero, that the group $\overline{\operatorname{Inn}}(A) / \overline{\operatorname{Inn}}_{0}(A)$ vanishes without $A$ being asymptotically abelian. Consider for example the case that $K_{1}(A)=0$ and

$$
K_{0}(A)=\mathbb{Q} \oplus \mathbb{Z}
$$

with strict ordering from the first coordinate and order unit $(1,0)$. Use Proposition 3.14 and Corollary 4.6.

The following proposition describes the topological structure of the quotient group $\overline{\operatorname{Inn}}(A) / \operatorname{Inn}_{0}(A)$. 
Proposition 4.9. The topological group $\overline{\operatorname{Inn}}(A) / \overline{\operatorname{Inn}}_{0}(A)$ is

(i) totally disconnected,

(ii) compact if and only if for every positive integer $n$ dividing [1] in $\mathrm{K}_{0}(A)$ the group

$$
\mathrm{K}_{1}(A) / n \mathrm{~K}_{1}(A)
$$

is finite,

(iii) locally compact if and only if there is a positive integer $n_{0}$ dividing [1] in $\mathrm{K}_{0}(A)$ such that

$$
n_{0} \mathrm{~K}_{1}(A) / n \mathrm{~K}_{1}(A)
$$

is finite for every positive integer $n$ that is divisible by $n_{0}$ and divides [1] in $\mathrm{K}_{0}(A)$, and that

(iv) discrete if and only if there is a positive integer $n_{0}$ dividing $[1]$ in $\mathrm{K}_{0}(A)$ such

$$
n \mathrm{~K}_{1}(A)=n_{0} \mathrm{~K}_{1}(A)
$$

for every positive integer $n$ that is divisible by $n_{0}$ and divides [1] in $\mathrm{K}_{0}(A)$.

In the last case,

$$
\overline{\operatorname{Inn}}(A) / \overline{\operatorname{Inn}}_{0}(A) \simeq \mathrm{K}_{1}(A) / n_{0} \mathrm{~K}_{1}(A) .
$$

If $\overline{\operatorname{Inn}}(A) / \overline{\operatorname{Inn}}_{0}(A)$ is not discrete, then it has no isolated points.

Proof. Each of these statements is easily proved for the inverse limit $\lim _{\leftarrow} \mathrm{K}_{1}(A) / n_{j} \mathrm{~K}_{1}(A)$, on using that the collection of subsets $\pi_{j}^{-1}(\{x\}), j \in \mathbb{N}$ and $x \in \mathrm{K}_{1}(A) / n_{j} \mathrm{~K}_{1}(A)$, is a basis of clopen sets for its topology. The proposition then follows by Theorem 4.5 .

Corollary 4.10. The group $\overline{\operatorname{Inn}}_{0}(A)$ is the connected component containing the identity of the automorphism group $\operatorname{Aut}(A)$ with the topology of pointwise convergence.

Proof. This follows immediately from Proposition 4.9(i) and Theorem 2.1, together with the fact that $\operatorname{Inn}_{0}(A)$ is connected.

From the properties of the group $\mathrm{U}_{0}(A)$ it is seen that $\operatorname{Inn}_{0}(A)$ is arc-connected and locally arc-connected. It is not true in general that every element of $\overline{\operatorname{Inn}}_{0}(A)$ is the limit of a path from $\operatorname{Inn}_{0}(A)$. Still, it does not seem unlikely that the following two questions have affirmative answers:

Is $\overline{\operatorname{Inn}}_{0}(A)$ locally arc-connected?

Is $\overline{\operatorname{Inn}}_{0}(A)$ arc-connected?

(Of course, an affirmative answer to the first question implies an affirmative answer to the second.)

4.11. Remarks. The conditions in (ii) and (iii) of Proposition 4.9 are satisfied if $\mathrm{K}_{1}(A)$ has finite rank, i.e. if

$$
\operatorname{dim}_{\mathbb{Q}} \mathrm{K}_{1}(A) \underset{\mathbb{Z}}{\otimes} \mathbb{Q}<\infty
$$


Proposition 4.9 also says that $\overline{\operatorname{Inn}}(A) / \overline{\operatorname{Inn}}_{0}(A)$ is either discrete, locally compact with no isolated points, or not locally compact. The examples below show that all three of these possibilities occur.

4.12. Examples.

(i) Let $B$ denote the Bunce-Deddens algebra with

$$
\mathrm{K}_{0}(B)=\mathbb{Z}\left[\frac{1}{2}\right], \quad \mathrm{K}_{1}(B)=\mathbb{Z},
$$

and order unit $1 \in \mathbb{Z}\left[\frac{1}{2}\right]$ (see also Example 2.4(iii)). Then $\overline{\operatorname{Inn}}(B) / \overline{\operatorname{Inn}}_{0}(B)$ is homeomorphic to the Cantor set. In fact, $\overline{\operatorname{Inn}}(B) / \overline{\operatorname{Inn}}_{0}(B)$ is isomorphic to the 2 -adic integers $\mathbb{Z}_{2}$. This can also be seen directly, as follows.

Write $B$ (in the standard way) as an inductive limit

$$
\mathrm{C}(\mathrm{T}) \rightarrow \mathrm{M}_{2}(\mathrm{C}(\mathrm{T})) \rightarrow \mathrm{M}_{4}(\mathrm{C}(\mathrm{T})) \rightarrow \cdots \rightarrow B,
$$

with each inclusion $\mathrm{C}(\mathrm{T}) \rightarrow \mathrm{M}_{2}(\mathrm{C}(\mathbb{T}))$ the standard twice around embedding. Consider the unitary $u \in C(\mathbb{T}), u(z)=z(z \in \mathbb{T})$, which is the generator of $K_{1}(B)=\mathbb{Z}$. Then (the image of) $u^{2^{n}}$ is central in $\mathrm{M}_{2^{n}}(\mathrm{C}(\mathbb{T}))$. It follows that the sequence $\left(u^{n_{j}}\right)$ is central in $A$ if (and only if) $n_{j}=2^{k_{j}} m_{j}$ and $k_{j} \rightarrow \infty$.

(ii) Let $G$ be a countable, torsion-free, abelian group, and let $n$ denote a positive integer. Choose an irrational number $\theta$, and let $A$ denote the inductive limit of circle algebras of real rank zero with K-theory

$$
\mathrm{K}_{0}(A)=\mathbb{Z}+\theta \mathbb{Z} \subseteq \mathbb{R}, \quad \mathrm{K}_{1}(A)=G,
$$

and with order unit $n \in \mathbb{Z}+\theta \mathbb{Z}$. Then

$$
\overline{\operatorname{Inn}}(A) / \overline{\operatorname{Inn}}_{0}(A)=G / n G
$$

(as a discrete group). The class of groups $G / n G$ attainable in this way contains of course all finite cyclic groups as well as some infinite groups. It contains the Klein four group $\mathbb{Z} / 2 \mathbb{Z} \oplus \mathbb{Z} / 2 \mathbb{Z}$, and also $\mathbb{Z} / 2 \mathbb{Z} \oplus \mathbb{Z} / 2 \mathbb{Z} \oplus \mathbb{Z} / 3 \mathbb{Z}$, but not $\mathbb{Z} / 2 \mathbb{Z} \oplus \mathbb{Z} / 4 \mathbb{Z}$.

(iii) Let $A$ denote the inductive limit of circle algebras of real rank zero with K-theory

$$
\mathrm{K}_{0}(A)=\mathbb{Z}\left[\frac{1}{2}\right] \subseteq \mathbb{R}, \quad \mathrm{K}_{1}(A)=\mathbb{Z}\left[\frac{1}{2}\right]^{(\infty)} \oplus \mathbb{Z},
$$

and order unit $3 \in \mathbb{Z}\left[\frac{1}{2}\right]$. Then

$$
\mathrm{K}_{1}(A) / 3 \mathrm{~K}_{1}(A)=(\mathbb{Z} / 3 \mathbb{Z})^{(\infty)}
$$

is infinite, and so $\overline{\operatorname{Inn}}(A) / \overline{\operatorname{Inn}}_{0}(A)$ is not compact. Since

$$
3 \mathrm{~K}_{1}(A) / 3 \cdot 2^{n} \mathrm{~K}_{1}(A)=\mathbb{Z} / 2^{n} \mathbb{Z}
$$

for all $n$, it follows that $\overline{\operatorname{Inn}}(A) / \overline{\operatorname{Inn}}_{0}(A)$ is locally compact but has no isolated points.

(iv) Let $A$ be given by the $\mathrm{K}$-theory

$$
\mathrm{K}_{0}(A)=\mathbb{Z}\left[\frac{1}{2}\right] \subseteq \mathbb{R}, \quad \mathrm{K}_{1}(A)=\mathbb{Z}^{(\infty)},
$$

and order unit $1 \in \mathbb{Z}\left[\frac{1}{2}\right]$. Then

$$
2^{n_{0}} \mathrm{~K}_{1}(A) / 2^{n} \mathrm{~K}_{1}(A)=\left(\mathbb{Z} / 2^{n-n_{0}} \mathbb{Z}\right)^{(\infty)}
$$

for all integers $0 \leqq n_{0}<n$. Hence $\overline{\operatorname{Inn}}(A) / \overline{\operatorname{Inn}}_{0}(A)$ is not locally compact in this case. 
4.13. Remarks and Questions. Some of the results about the group $\overline{\operatorname{Inn}}(A) / \overline{\operatorname{Inn}_{0}}(A)$ proved in this section for simple inductive limits of circle algebras of real rank zero may hold much more generally. Some of them perhaps even for all unital $\mathrm{C}^{*}$ algebras.

If $A$ is such that the map $\mathrm{U}(A) \rightarrow \mathrm{K}_{1}(A)$ is surjective, and its kernel is contained in the kernel of $\mathrm{U}(A) \rightarrow \overline{\operatorname{Inn}}(A) / \overline{\operatorname{Inn}_{0}}(A)$, then $\mathrm{K}_{1}(A) \rightarrow \overline{\operatorname{Inn}}(A) / \overline{\operatorname{Inn}_{0}}(A)$ is defined; cf. Lemma 4.1. In 4.3 let the condition $n$ divides [1] in $\mathrm{K}_{0}(A)$ be replaced with the condition $A$ contains a unital subalgebra isomorphic to $\mathrm{M}_{n}$. Then the " $\|$ " part of the proof of the condition Theorem 4.5 remains valid, and we get a continuous surjective homomorphism

$$
\overline{\operatorname{Inn}}(A) / \overline{\operatorname{Inn}}_{0}(A) \rightarrow \lim \mathrm{K}_{1}(A) / n_{j} \mathrm{~K}_{1}(A) .
$$

However, this map is not injective in general. Consider for example $A=\mathrm{M}_{2}\left(\mathrm{C}\left(\mathrm{T}^{3}\right)\right)$. For that algebra,

$$
\begin{aligned}
\overline{\operatorname{Inn}}(A) / \overline{\operatorname{Inn}_{0}}(A) & =(\mathbb{Z} / 2 \mathbb{Z})^{3} \oplus \mathbb{Z}, \\
\lim \mathrm{K}_{1}(A) / n_{j} \mathrm{~K}_{1}(A) & =\mathrm{K}_{1}(A) / 2 \mathrm{~K}_{1}(A)=(\mathbb{Z} / 2 \mathbb{Z})^{4} .
\end{aligned}
$$

It is essential in this example that certain $\mathrm{K}_{1}$ classes do not live in some small corners of the algebra. That phenomenon will disappear if the algebra has stable rank one, and possibly also if the algebra is simple. Consider, as another example, the simple $C^{*}$-algebra $\mathrm{C}_{r}^{*}\left(F_{2}\right)$, the reduced $\mathrm{C}^{*}$-algebra of the free group on two generators. Every central sequence in $\mathrm{C}_{r}^{*}\left(F_{2}\right)$ is trivial (see [P]). Hence, if $u \in \mathrm{C}_{r}^{*}\left(F_{2}\right)$ is unitary, then $\mathrm{Ad} u \in \overline{\operatorname{Inn}}_{0}\left(\mathrm{C}_{r}^{*}\left(F_{2}\right)\right)$ only if $u$ is in the connected component of 1 (cf. Lemma 4.2). It follows that the group

$$
\overline{\operatorname{Inn}}\left(C_{r}^{*}\left(F_{2}\right)\right) / \overline{\operatorname{Inn}}_{0}\left(C_{r}^{*}\left(F_{2}\right)\right)
$$

is non-trivial. On the other hand, by [PV], the right side of $(*)$ is zero.

When is the map (*) an isomorphism?

The inverse limit $\lim _{\leftarrow} \mathrm{K}_{1}(A) / n_{j} \mathrm{~K}_{1}(A)$ is always totally disconnected, so if $(*)$ is injective, then $\overline{\operatorname{Inn}}(A) / \overline{\operatorname{Inn}}_{0}(A)$ is also totally disconnected. $A ?$

Is $\overline{\operatorname{Inn}}(A) / \overline{\operatorname{Inn}}_{0}(A)$ totally disconnected for every (separable) unital $\mathrm{C}^{*}$-algebra

\section{References}

[Bk] Brenken, B.: Representations and automorphisms of the irrational rotation algebra. Pacific J. Math. 2, 257-282 (1984)

[BBEK] Blackadar, B., Bratteli, O., Elliott, G.A., Kumjian, A.: Reduction of real rank in inductive limit $C^{*}$-algebras. Math. Ann. 292, 111-126 (1992)

[BKR] Blackadar, B., Kumjian, A., Rørdam, M.: Approximately central matrix units and the structure of non-commutative tori. K-Theory 6, 267-284 (1992)

[Bw] Brown, L.: Stable isomorphism of heredity subalgebras of $\mathrm{C}^{*}$-algebras. Pacific J. Math. 71, 335-348 (1977)

[C] Cuntz, J.: K-theory for certain C*-algebras. Ann. Math. 113, 181-197 (1981)

[E1] Elliott, G.A.: Dimension groups with torsion. Int. J. Math. 1, 361-380 (1990) 
[E2] Elliott, G.A.: On the classification of $\mathrm{C}^{*}$-algebras of real rank zero. J. Reine Angew. Math. (to appear)

[EE] Elliott, G.A., Evans, D.E.: The structure of the irrational rotation $\mathrm{C}^{*}$-algebra. Ann. Math. (to appear)

[EG] Elliott, G.A., Gong, G.: On inductive limits of matrix algebras over the two-torus. Preprint.

[HP] de la Harpe, P., Skandalis, G.: Sur la simplicité essentielle du groupe des invertibles du groupe unitaire dans une $C^{*}$-algèbre simple. J. Funct. Anal. 62, 354-378 (1985)

[K] Kumjian, A.: An involutive automorphism of the Bunce-Deddens algebra. C.R. Math. Rep. Acad. Sci. Canada 10, 217-218 (1988)

[P] Powers, R.T.: Simplicity of the $\mathrm{C}^{*}$-algebra associated with the free group on two generators. Duke Math. J. 42, 151-156 (1975)

[PV] Pimsner, M., Voiculescu, D.: K-groups of reduced crossed products by free groups. J. Operator Theory 8, 131-156 (1982)

[R] Rørdam, M.: On the structure of simple $\mathrm{C}^{*}$-algebras tensored with a UHF-algebra. J. Funct. Anal. 100, 1-17 (1991)

[T] Thomsen, K.: Finite sums and products of commutators in inductive limit $\mathrm{C}^{*}$-algebras. Preprint

[W] Watatani, Y.: Toral automorphisms on irrational rotation algebras. Math. Japonica 26, 479-484 (1981)

[Z] Zhang, S.: A Riesz decomposition property and ideal structure of multiplier algebras. J. Operator Theory (to appear)

Communicated by H. Araki 